\title{
A pairwise surface contact equation of state: COSMO-SAC-Phi
}

\author{
Rafael de P. Soares*, Luis F. Baladão, Paula B. Staudt \\ Virtual Laboratory for Properties Prediction (LVPP), Chemical Engineering Department, Federal University of Rio Grande do Sul, Rua Ramiro Barcelos, 2777, \\ CEP 90035-007, Porto Alegre, RS, Brazil
}

\section{A R T I C L E I N F O}

\section{Article history:}

Received 1 December 2018

Received in revised form 14 January 2019

Accepted 15 January 2019

Available online 22 January 2019

Keywords:

COSMO-RS

COSMO-SAC

F-SAC

Association

Lattice-fluid

\begin{abstract}
A B S T R A C T
In this work a new method for inclusion of pressure effects in COSMO-type activity coefficient models is proposed. The extension consists in the direct combination of COSMO-SAC and lattice-fluid ideas by the inclusion of free volume in form of holes. The effort when computing pressure (given temperature, volume, and mole numbers) with the proposed model is similar to the cost for computing activity coefficients with any COSMO-type implementation. For given pressure, computational cost increases since an iterative method is needed. This concept was tested for representative substances and mixtures, ranging from light gases to molecules with up to 10 carbons. The proposed model was able to correlate experimental data of saturation pressure and saturated liquid volume of pure substances with deviations of $1.16 \%$ and $1.59 \%$, respectively. In mixture vapor-liquid equilibria predictions, the resulting model was superior to Soave-Redlich-Kwong with Mathias-Copeman $\alpha$-function and the classic van der Waals mixing rule in almost all cases tested and similar to PSRK method, from low pressures to over 100 bar. Good predictions of liquid-liquid equilibrium were also observed, performing similarly to UNIFAC-LLE, with improved responses at high temperatures and pressures.
\end{abstract}

() 2019 Elsevier B.V. All rights reserved.

\section{Introduction}

Thermodynamic models for mixtures with high predictive power are of great interest for both academia and industry. The COSMO-RS [1] model and others based on its surface contact theory, such as COSMO-SAC [2] and F-SAC [3,4], are interesting alternatives for mixture behavior prediction when limited experimental data is available.

Although these models are very successful, they describe incompressible liquids only, failing to represent effects of pressure on thermodynamic properties, as is schematically shown in Fig. 1.

The basic assumption in COSMO-type models is that molecules exist in a fixed volume cavity completely surrounded by other molecules, with no empty spaces between them. In Fig. 1 there are actually empty spaces between the molecules, just because of our inability to draw their surfaces completely surrounded by other molecules.

Some approaches can be found in the literature attempting to combine COSMO-based models with equations of state to represent compressible fluids. For instance, Panayiotou [5,6] developed an

\footnotetext{
* Corresponding author.

E-mail address: rafael.pelegrini@ufrgs.br (R. de P. Soares).
}

equation of state based, in part, on COSMO-RS method. The author calculated chemical potentials of interacting surfaces in terms of the so called nonrandomness factors. The method was applied for some normal alkanes and polyethylene, later the author also computed solvation/hydration properties [6,7]. By adding vacancies to the solvent surface area, Shimoyama and Iwai [8] predicted solid solubilities in supercritical carbon dioxide with average deviations smaller than one logarithmic unit with the so called COSMO-vac model. However, the method still computes activity coefficients only and cannot be seen as a complete equation of state. More recently, Costa et al. $[9,10]$ developed an equation of state called $\sigma$ MTC, an extension of Mattedi-Tavares-Castier equation [11] which combines the sigma-profile from COSMO computations with the generalized van der Waals theory.

Another approach investigated in the literature is the combination of COSMO-SAC with cubic equations of state by means of mixing rules. For instance, Lee and Lin [12] combined the PengRobinson (PR) equation of state with COSMO-SAC model using the Wong-Sandler mixing rule ( $\mathrm{PR}+\mathrm{WS}+\mathrm{COSMO}-\mathrm{SAC})$ to predict VLE in binary systems, including associative and non-associative mixtures. Hsieh and Lin [13] proposed a methodology for calculating cubic equations of state parameters using concepts from the solvation theory of Ben-Naim [14]. Later, the PR+COSMO-SAC model of Hsieh and Lin $[15,16]$ was used to predict vapor-liquid 
COSMO surface for water, its core volume $b_{i}$ is constant
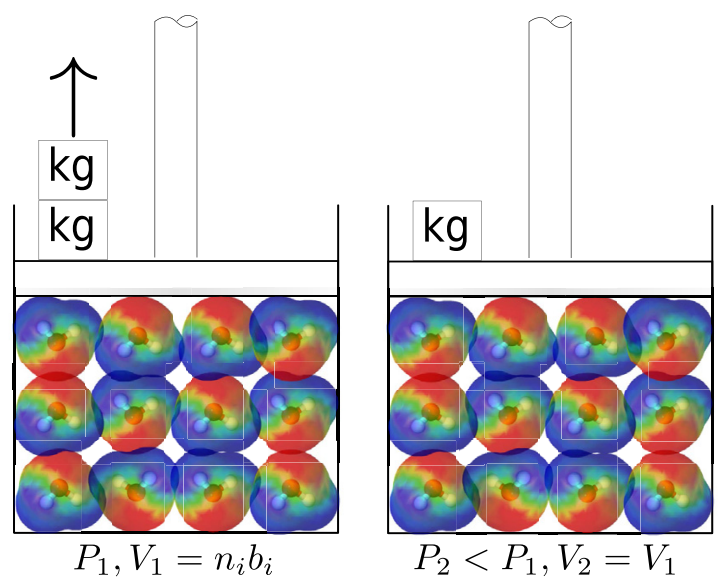

Fig. 1. COSMO-SAC schematic representation - the volume should be constant regardless of the pressure and no empty spaces are considered.

equilibria of pure fluids and mixtures. Staudt and Soares [17] also combined cubic equations of state with COSMO-SAC, using the so called Self-Consistent Mixing Rule (SCMR). More recently, Wang et al. [18] used SCMR when modeling polymer/gas mixtures based on COSMO-SAC, proposing a modified version, mSCMR. The authors concluded that mSCMR is superior to Wong-Sandler [19] and MHV1 [20] mixing rules for the cases studied.

In the present work, an extension of COSMO-like models to include pressure effects is developed by directly combining latticefluid ideas with COSMO-RS theory. The proposed model can also be seen as a perturbation model, with a simple fluid as reference and a COSMO-based perturbation term. The resulting method is straightforward and the computational cost (for given temperature, volume, and mole numbers) is the same required by any COSMOtype implementation. Since the proposed method was tested with COSMO-SAC model and uses its notation, the resulting model is referred hereinafter as COSMO-SAC-Phi, or CSP for short.

\section{The COSMO-SAC-Phi method}

In this work, a new model inspired by lattice-fluid theory is proposed, called COSMO-SAC-Phi. In this model, molecule volume is still constant but pressure effects and volume expansion are represented by the presence of holes (also of fixed volume), as depicted in Fig. 2. Again, there should be no empty spaces and all surfaces should be in pairwise contact.

As in any perturbation model [21], we describe pressure as the sum of two contributions:

$P=P_{R}+P_{A}$

where $P_{R}$ is the reference fluid pressure (in this work also including ideal gas contribution) and $P_{A}$ is the pressure due to attractive forces (perturbation, usually negative).

\subsection{Attractive contribution by a pseudo-mixture}

The main contribution of the present work is the proposal of a new attraction (perturbation) term by directly combining COSMORS theory with lattice-fluid ideas [22,23]. However, there is no need

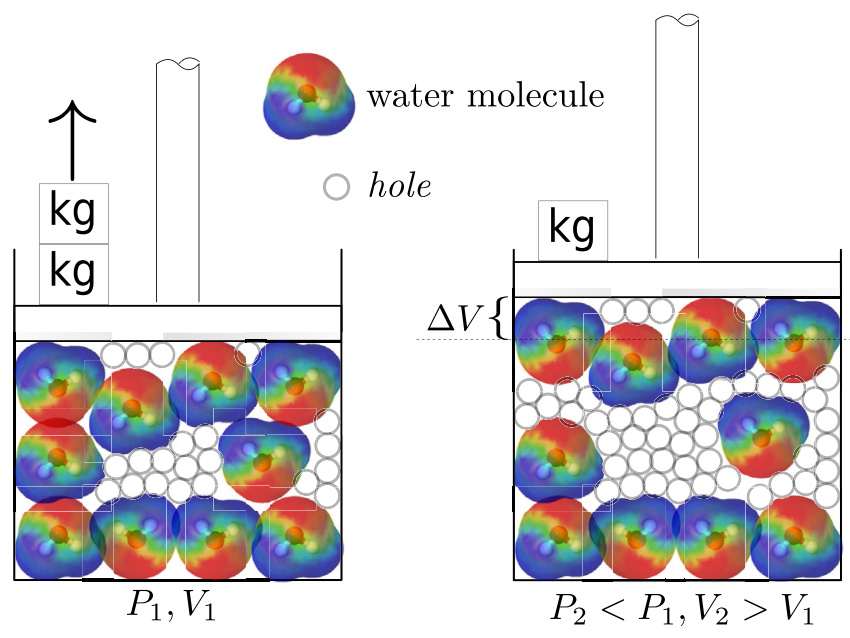

Fig. 2. COSMO-SAC-Phi schematic representation - volume expansions are described by the addition of holes of fixed volume.

to actually define a lattice and there is no explicit coordination number (i.e., a number of nearest neighbors interacting with a given molecule). When compared to NRCosmo [6] or $\sigma$-MTC [9,10], the main difference is that these methods combine COSMO-based information with existing equations of state, while the proposed method directly extends COSMO-SAC to become an equation of state. When compared to COSMO-vac [8], the idea of vacancies is similar to the method proposed in this work. However, COSMO-vac can only compute activity coefficients of a solute diluted in a supercritical phase, while the proposed method is a full equation of state.

The first fundamental point of this proposal is that even pure substances are schematically represented as a pseudo-mixture, like in Fig. 2. For a real mixture its composition is described by a mole amount vector $\mathbf{n}=\left[n_{1}, n_{2}, \ldots, n_{i}, \ldots, n_{N}\right]$ and the corresponding pseudo-mixture, by the combination of the real mole amount vector and the amount of holes $n_{h}: \tilde{\mathbf{n}}=\left[\mathbf{n}, n_{h}\right]$.

Assuming that there are no empty spaces (other than the holes) in mixture, we can represent the system total volume by:

$V=\sum_{i} n_{i} b_{i}+n_{h} b_{h}$

where $b_{i}$ is the molar cavity volume of species $i$ and $b_{h}$ is the molar cavity volume of a hole. Eq. (2) is obviously a crude simplification because if we define a form for the holes (for instance a sphere) there will still be volume not occupied either by molecules or holes in mixture. In this sense, as a first attempt to describe pressure effects, the holes do not have a specific form, but should occupy all free volume available.

As depicted in Fig. 2, we relate pressure-volume effects to the inclusion or removal of holes. This feature is not present in COSMO$\mathrm{RS}$, COSMO-SAC, or F-SAC models. For a given total volume and mole amount vector, Eq. (2) can be used to compute the hole mole amount in the system:

$n_{h}=\frac{1}{b_{h}}\left(V-\sum_{i} n_{i} b_{i}\right)$

For the calculation of attractive pressure $P_{A}$, a classic thermodynamic relation can be used: 
$P_{A}=-\left(\frac{\partial A_{A}^{r}}{\partial V}\right)_{T, \mathbf{n}}$

where $A_{A}^{r}$ is the residual Helmholtz energy of the attractive contribution. To simplify the notation, we will drop the subscript $A$ from here forward.

Considering the pseudo-mixture scheme of Fig. 2, we can derive the required Helmholtz derivative by:

$\left(\frac{\partial A^{r}}{\partial V}\right)_{T, \mathbf{n}}=\left(\frac{\partial \tilde{A}^{r}}{\partial n_{h}}\right)_{T, \mathbf{n}}\left(\frac{\partial n_{h}}{\partial V}\right)_{T, \mathbf{n}}$

where $\tilde{A}^{r}$ is the residual Helmholtz energy of pseudo-mixture and the last term of Eq. (5) is directly obtained by deriving Eq. (3):

$\left(\frac{\partial n_{h}}{\partial V}\right)_{T, \mathbf{n}}=\frac{1}{b_{h}}$

Finally, we define the residual chemical potential of the holes in the pseudo-mixture as:

$\tilde{\mu}_{h}^{r} \equiv\left(\frac{\partial \tilde{A}^{r}}{\partial n_{h}}\right)_{T, n_{j \neq h}}=\left(\frac{\partial \tilde{A}^{r}}{\partial n_{h}}\right)_{T, \mathbf{n}}$

leading to an elegant result:

$P_{A}=-\left(\frac{\partial A^{r}}{\partial V}\right)_{T, \mathbf{n}}=-\frac{\tilde{\mu}_{h}^{r}}{b_{h}}$

The holes chemical potential $\tilde{\mu}_{h}$ (not residual) in pseudomixture, and the other components chemical potential $\tilde{\mu}_{i}$, are computed as usual with models such as COSMO-RS, COSMO-SAC, or F-SAC. To obtain the residual version of these chemical potentials, we present a simple approach.

Considering COSMO-SAC notation, we discretize a molecule surface in several segments, and the activity coefficient of a segment $\Gamma_{m}$ is given by:

$\ln \Gamma_{m}=-\ln \left\{\sum_{n} p_{n} \Gamma_{n} \exp \left[\frac{-\Delta W_{m, n}}{R T}\right]\right\}$

where $\Delta W_{m, n}$ is the interaction energy between segments $m$ and $n$ and $p_{n}$ is the probability of finding segment $n$. In the proposed model, both holes and molecules are always considered in Eq. (9). As stated by Lin and Sandler [2], the logarithm of the activity coefficient of a segment $\ln \Gamma_{m}$ is actually a difference of chemical potentials. More precisely, $\ln \Gamma_{m}$ is the difference in chemical potential between the segment inserted in the actual mixture with respect to the segment inserted into a fluid of identical neutral segments.

To compute the residual contribution, and then respect the ideal gas limit, the neutral segment fluid reference should be replaced by the usual reference of an ideal gas. This can be accomplished by the following subtraction:

$\ln \Gamma_{m}^{r}=\ln \Gamma_{m}-\ln \Gamma_{m}^{\mathrm{IG}}$

where $\ln \Gamma_{m}^{r}$ becomes the residual chemical potential of segment $m$.

The chemical potential of the segment in an ideal gas $\ln \Gamma_{m}^{\mathrm{IG}}$ can be calculated with Eq. (9) evaluated at infinite molar volume limit, at the same temperature. This can be accomplished by making the pseudo-mixture mole amount vector as:
$\tilde{\mathbf{n}}^{\mathrm{IG}}=\left[\mathbf{n}=0, n_{h}=1\right]=[0,0, \ldots, 1]$

Finally, with the residual chemical potential of each segment, we can calculate the residual chemical potential of a given compound (or hole) by summing its segments:

$\tilde{\mu}_{i}^{r}=R T \sum_{m \in i}\left(\frac{Q_{m}}{a_{\mathrm{eff}}}\right) \ln \Gamma_{m}^{r}$

$\tilde{\mu}_{h}^{r}=R T \sum_{m \in h}\left(\frac{Q_{m}}{a_{\text {eff }}}\right) \ln \Gamma_{m}^{r}$

where $a_{\mathrm{eff}}=\pi r_{a v}^{2}$ is the standard surface area segment and $r_{a v}$ is the averaging radius; $Q_{m}$ is the surface area of segment $m$.

\subsection{Fugacity coefficients}

Fugacity coefficients can be computed by [24]:

$\ln \widehat{\phi}_{i}=\frac{1}{R T}\left(\frac{\partial A^{r}}{\partial n_{i}}\right)_{T, V, n_{j}}-\ln Z$

where $Z \equiv P V / N R T$ is the compressibility factor.

Since we are considering that interactions come from a sum of repulsive and attractive forces, residual Helmholtz energy is $A^{r}=$ $A_{R}^{r}+A_{A}^{r}$. Again, we will describe only the attractive contribution in this section and will drop the subscript to simplify the notation.

In order to evaluate the Helmholtz partial derivative of Eq. (14) for a COSMO-SAC-Phi pseudo-mixture, an increase in the amount $n_{i}$ should cost a reduction of holes amount $n_{h}$ to keep a constant total volume $V$. This is illustrated in Fig. 3 and can be transcribed to:

$\left(\frac{\partial A^{r}}{\partial n_{i}}\right)_{T, V, n_{j}}=\left(\frac{\partial \tilde{A}^{r}}{\partial n_{i}}\right)_{T, V, n_{j}, n_{h}}+\left(\frac{\partial \tilde{A}^{r}}{\partial n_{h}}\right)_{T, V, \mathbf{n}}\left(\frac{\partial n_{h}}{\partial n_{i}}\right)_{T, V, n_{j}}$

where the last term on the right hand side is obtained by deriving Eq. (3) (with constant $b_{h}$ ):

$\left(\frac{\partial n_{h}}{\partial n_{i}}\right)_{T, V, n_{j}}=-\frac{b_{i}}{b_{h}}$
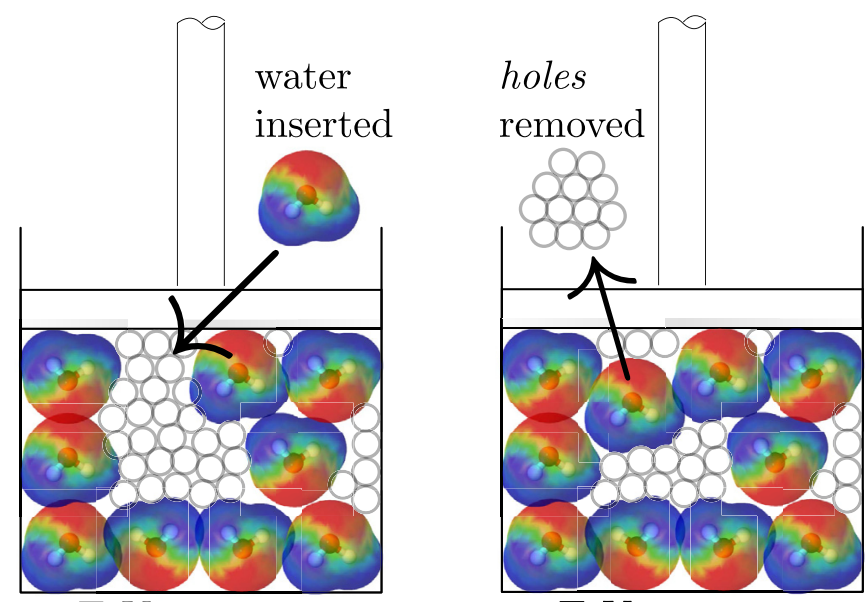

$T, V, n_{i, 1}, n_{h, 1}$

$T, V, n_{i, 2}, n_{h, 2}$

Fig. 3. COSMO-SAC-Phi illustration of constant total volume $V$ system: in order to introduce a new molecule holes have to be removed. 
Combining Eqs. (15) and (16) with the chemical potential in Eq. (7), we obtain another elegant result:

$\left(\frac{\partial A^{r}}{\partial n_{i}}\right)_{T, V, n_{j}}=\tilde{\mu}_{i}^{r}-\tilde{\mu}_{h}^{r} \frac{b_{i}}{b_{h}}$

Eq. (17) can be applied to obtain fugacity coefficients of pure substances and fugacity coefficients of substances in mixtures.

If the hole volume $b_{h}$ (representing free volume) is not the same for every compound in mixture, a mixing rule is used:

$b_{h}=\sum_{i} \frac{n_{i}}{N} b_{h, i}$

where $N=\sum_{i} n_{i}$; and $b_{h, i}$ is the hole volume for species $i$. Finally, the expression for Helmholtz partial derivative of Eq. (14) would be:

$$
\left(\frac{\partial A^{r}}{\partial n_{i}}\right)_{T, V, n_{j}}=\tilde{\mu}_{i}^{r}-\tilde{\mu}_{h}^{r}\left(\frac{b_{i}}{b_{h}}+\frac{n_{h}}{N}\left(b_{h, i} / b_{h}-1\right)\right)
$$

\section{Additional assumptions and computational aspects}

For actual computations with the proposed method some additional assumptions are needed. In this section we describe these additional assumptions and give more details in pressure and fugacity coefficient calculations.

\subsection{Repulsive forces}

As a first approximation, in the present work a simple hardsphere model was chosen to represent repulsive forces (reference fluid):

$P_{R}=\frac{N R T}{V-\sum_{i} n_{i} b_{i}}$

where $N=\sum_{i} n_{i} ; R$ is the universal gas constant; $T$ is the temperature; and $b_{i}$ is the hard-sphere volume of species $i$.

The hole amount $n_{h}$ and its molar volume $b_{h}$ should not be included in the computation of Eq. (20). More sophisticated representations, such as the Carnahan and Starling [25] or the more recent developments present in SAFT models [26,27] may be used in future studies.

\subsection{Cavity volumes and areas}

Molecule volumes $b_{i}$ are necessary in the proposed model, e.g. Eqs. (2) and (20). The COSMO cavity volumes could be considered as a totally predictive alternative for $b_{i}$. However, good quantitative results were not observed directly using these values. Thus, in the present work, values of $b_{i}$ were optimized with pure compound experimental data (vapor pressure and saturated liquid volume). COSMO cavity surface areas and apparent surface charge densities were not optimized. COSMO cavity volumes calculated as described by Ferrarini et al. [28] were used as initial guesses in the estimation problem.

The area of one hole was assumed to be a universal constant, corresponding to the area of $1 \AA$ A radius sphere $\left(12.57 \AA^{2}\right)$. A much smaller hole surface area would be inconsistent with the underlying COSMO-SAC contact surface area $[2,28,29]$. The hole cavity volume $b_{h}$ (representing free volume) was optimized individually for each substance. A universal value for $b_{h}$ was not possible for the precise representation of saturated liquid volumes and equilibrium pressures. Substances more tightly packed, as water and ammonia, required smaller values of $b_{h}$ (see Table 1 and Fig. 5). For larger molecules a nearly constant value of $b_{h}$ was observed and a constant value around $16 \AA^{3}$ could be used.

\subsection{Segment interaction energies}

For segment interaction energies, a formulation including dispersion forces, similar to the one developed by Flôres et al. [30], was considered:

$\Delta W_{m, n}=\frac{\alpha^{\prime}\left(\sigma_{m}+\sigma_{n}\right)^{2}}{2}+\frac{E_{m, n}^{\mathrm{HB}}}{2}+\frac{E_{m, n}^{\mathrm{Disp}}}{2}$

where $\sigma_{m}$ is the apparent surface charge density of segment $m ; \alpha^{\prime}$ is a constant; $E_{m, n}^{\mathrm{HB}}$ is the hydrogen bond (HB) formation energy; and $E_{m, n}^{\text {Disp }}$ is the dispersion contribution.

The required COSMO apparent surface charge densities were taken from the open source LVPP-sigma profile database [28], freely available at https://github.com/lvpp/sigma. The COSMO-SAC universal constants and HB energies were taken from the GMHB1808 parametrization, also freely available on that github repository. This parametrization assumes that only hydrogen atoms bonded to oxygen require additional HB energy (other than the electrostatic contribution) and there is no dispersion correction. It is also noteworthy that only the residual contribution is necessary in the proposed model since combinatorial contribution should come from the repulsive term (reference fluid).

Dispersion effects were computed by a simple combining rule:

$E_{m, n}^{\text {Disp }}=-\sqrt{\delta_{m} \delta_{n}}$

being the segment dispersion $\delta_{m}$ temperature-dependent, with a decay inspired by the one used in PC-SAFT models for temperaturedependent segment diameters [27]:

$\delta_{m}=\delta_{m}^{0}\left(1-\exp \left(-\delta_{m}^{T} / T\right)\right)$

where $\delta_{m}^{0}$ and $\delta_{m}^{T}$ were assumed to be per compound parameters $\left(\delta_{m}=\delta_{i}\right)$ and, in the present work, were adjusted with pure compound experimental data.

For holes, the surface charge densities were assumed zero as well as the parameter $\delta_{m}^{0}$. As a consequence, no dispersion effects occur for holes and $\delta_{m}^{T}$ becomes irrelevant.

More sophisticated alternatives for dispersion contribution, with stronger predictive power, could be investigated in future works. In the opposite direction, the inclusion of binary interaction parameters in Eq. (22) is also possible, resulting in $E_{i, j}^{\text {Disp }}=-\sqrt{\delta_{i} \delta_{j}}\left(1-k_{i j}\right)$ with $k_{i j}$ fitted using binary equilibrium data.

\subsection{Pressure and fugacity coefficient computation}

For given temperature, total volume, and mole amounts, pressure can be computed with COSMO-SAC-Phi following the flowchart presented in Fig. 4.

In principle, flowchart in Fig. 4 can be applied to introduce pressure-volume effects in any COSMO-based implementation. As can be seen in this figure, repulsion pressure is computed with a single step: R1. For attraction pressure, multiple steps are necessary. The first step is to compute ideal gas segment potentials in A1. For this, the pseudo-mixture mole vector $\tilde{\mathbf{n}}$ is set as pure holes, with molecules infinitely diluted in it. In A1 and A3 Eq. (9) needs to be solved. This is actually the solution of a system of equations 
Table 1

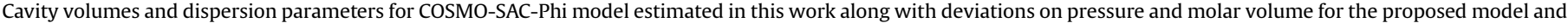
SRK-MC, with liquid volumes corrected by volume translation (VT).

\begin{tabular}{|c|c|c|c|c|c|c|c|c|c|}
\hline \multirow[t]{2}{*}{ Family } & \multirow[t]{2}{*}{ Compound } & \multirow{2}{*}{$\frac{b_{i}}{\mathrm{~A}^{3}}$} & \multirow{2}{*}{$\frac{\delta_{m}^{0}}{\mathrm{kcal} / \mathrm{mol}}$} & \multirow{2}{*}{$\frac{\delta_{m}^{T}}{10^{2} \mathrm{~K}}$} & \multirow{2}{*}{$\frac{b_{h, i}}{\mathrm{~A}^{3}}$} & \multicolumn{2}{|c|}{ COSMO-SAC-Phi } & \multicolumn{2}{|c|}{$\mathrm{SRK}-\mathrm{MC}+\mathrm{VT}$} \\
\hline & & & & & & $\mathrm{RD}_{P} \%$ & $\mathrm{RD}_{v} \%$ & $\mathrm{RD}_{P} \%$ & $\mathrm{RD}_{v} \%$ \\
\hline \multirow{10}{*}{$\begin{array}{l}\text { Saturated } \\
\text { hydrocarbon }\end{array}$} & methane & 49.70 & 0.526 & 3.050 & 11.63 & 1.78 & 1.20 & 1.47 & 3.16 \\
\hline & ethane & $72.9 \mathrm{G}$ & 0.709 & 3.479 & 13.41 & 1.45 & 1.00 & 0.23 & 3.10 \\
\hline & propane & 98.74 & 0.755 & 3.556 & 15.40 & 1.13 & 1.25 & 0.39 & 3.97 \\
\hline & $n$-butane & 126.40 & 0.778 & 3.641 & 16.53 & 0.50 & 0.66 & 0.34 & 3.17 \\
\hline & $n$-pentane & 154.73 & 0.804 & 3.558 & 17.41 & 0.26 & 0.44 & 0.21 & 3.14 \\
\hline & 2-methylpentane & 197.54 & 0.742 & 4.660 & 14.06 & 2.46 & 2.82 & 1.87 & 1.20 \\
\hline & $n$ - hexane & 190.84 & 0.813 & 3.614 & 16.61 & 0.55 & 1.60 & 1.17 & 3.73 \\
\hline & n-octane & 251.86 & 0.874 & 3.210 & 17.55 & 0.92 & 2.50 & 3.36 & 4.09 \\
\hline & n-decane & 324.26 & 0.913 & 3.025 & 16.70 & 2.01 & 4.48 & 1.38 & 4.88 \\
\hline & cyclohexane & 151.93 & 0.946 & 4.320 & 18.28 & 0.74 & 1.02 & 0.40 & 3.36 \\
\hline \multirow{4}{*}{$\begin{array}{l}\text { Unsaturated } \\
\text { hydrocarbon }\end{array}$} & ethylene & 66.80 & 0.554 & 4.222 & 11.99 & 1.27 & 0.54 & 0.72 & 2.73 \\
\hline & 1-butene & 116.56 & 0.730 & 3.848 & 16.17 & 0.50 & 0.64 & 2.63 & 3.17 \\
\hline & 1-hexene & 183.45 & 0.799 & 3.724 & 15.80 & 1.79 & 2.29 & 1.82 & 5.32 \\
\hline & cyclohexene & 139.09 & 0.928 & 4.749 & 16.54 & 1.33 & 0.66 & 8.40 & 18.21 \\
\hline \multirow[t]{2}{*}{ Aromatic } & benzene & 125.31 & 0.909 & 4.895 & 16.35 & 0.82 & 0.52 & 0.50 & 3.79 \\
\hline & toluene & 151.86 & 0.931 & 4.391 & 18.09 & 1.15 & 1.07 & 0.89 & 3.99 \\
\hline \multirow[t]{2}{*}{ Ketone } & acetone & 100.46 & 0.846 & 4.138 & 15.41 & 0.68 & 0.62 & 1.16 & 5.66 \\
\hline & methyl ethyl ketone & 130.12 & 0.859 & 4.345 & 14.08 & 0.62 & 0.98 & 1.58 & 1.03 \\
\hline \multirow[t]{3}{*}{ Ether } & dimethyl ether & 81.63 & 0.784 & 3.677 & 13.58 & 0.84 & 1.13 & 2.25 & 15.98 \\
\hline & diethyl ether & 140.12 & 0.807 & 3.365 & 16.22 & 0.35 & 0.42 & 0.76 & 4.58 \\
\hline & tetrahydrofuran & 111.14 & 0.972 & 4.302 & 16.03 & 1.45 & 0.91 & 1.12 & 4.59 \\
\hline \multirow[t]{2}{*}{ Ester } & methyl acetate & 109.55 & 0.783 & 3.557 & 14.51 & 0.47 & 0.47 & 2.58 & 4.42 \\
\hline & ethyl acetate & 141.41 & 0.821 & 3.276 & 15.35 & 0.73 & 1.42 & 1.05 & 4.11 \\
\hline \multirow[t]{2}{*}{ Organic halide } & chloroform & 111.26 & 0.921 & 4.710 & 13.31 & 1.04 & 3.22 & 1.16 & 12.19 \\
\hline & carbon tetrachloride & 135.13 & 0.885 & 4.672 & 16.18 & 0.79 & 1.08 & 0.91 & 3.62 \\
\hline \multirow[t]{4}{*}{ Alcohol } & methanol & 59.73 & 1.080 & 3.523 & 7.85 & 1.06 & 2.37 & 0.84 & 6.94 \\
\hline & ethanol & 92.00 & 1.208 & 2.667 & 7.84 & 1.80 & 4.04 & 2.78 & 5.74 \\
\hline & n-butanol & 148.39 & 1.484 & 1.948 & 10.81 & 1.42 & 5.12 & 1.99 & 4.97 \\
\hline & n-pent anol & 167.02 & 1.702 & 1.530 & 14.10 & 2.30 & 2.16 & 3.75 & 3.49 \\
\hline \multirow[t]{2}{*}{ Amine } & methylamine & 53.18 & 0.978 & 3.216 & 11.84 & 1.49 & 4.20 & 1.91 & 17.86 \\
\hline & triethylamine & 188.29 & 0.885 & 3.009 & 21.72 & 1.23 & 1.33 & 3.52 & 3.19 \\
\hline \multirow[t]{3}{*}{ Gases } & nitrogen & 45.11 & 0.320 & 2.100 & 10.29 & 1.83 & 0.97 & 1.89 & 3.26 \\
\hline & carbon dioxide & 46.45 & 0.400 & 4.431 & 9.07 & 0.17 & 0.17 & 0.77 & 5.77 \\
\hline & hydrogen sulfide & 46.88 & 0.755 & 5.717 & 10.49 & 1.56 & 0.91 & 1.24 & 5.33 \\
\hline \multirow[t]{3}{*}{ Other } & ammonia & 29.22 & 0.684 & 3.693 & 7.54 & 2.32 & 2.17 & 1.54 & 6.48 \\
\hline & water & 24.98 & 1.103 & 4.934 & 6.67 & 0.87 & 0.82 & 0.62 & 9.00 \\
\hline & & & & average & & 1.16 & 1.59 & 1.64 & 5.53 \\
\hline
\end{tabular}

(characteristic of COSMO-based models), usually accomplished by successive substitution. These steps are the most computational intensive, with the same cost required by any COSMO-based model when computing activity coefficients of a mixture. Efficient implementations can explore the fact that the quantity $\ln \Gamma_{m}^{\mathrm{IG}}$ depends only on temperature, and execute A1 only if temperature have changed.

In A2 the mole amount of hole $n_{h}$ is computed and then used to set the pseudo-mixture mole number vector. With $\tilde{\mathbf{n}}$ updated, Eq. (9) can be solved again. With the difference between the segment chemical potentials and the ideal gas ones, the residual segment chemical potentials are obtained (A4). Then, it is simple to obtain the pressure following A5-A7.

Interestingly, all quantities required to the computation of fugacity coefficients with Eq. (17) or Eq. (19) are readily available after a pressure computation as described in Fig. 4. Thus, no appreciable computational burden is introduced to obtain the fugacity coefficients.

For given $T, P, \mathbf{n}$ (for instance in phase equilibria calculations), an outer loop is necessary to solve the volume as a function of pressure, increasing significantly the computational cost. However, the A1 step can be computed only once, ameliorating the problem. In this work a secant method was used to solve the volume. For vapor phases the volume initial guess was computed assuming an ideal gas, and for liquid phases the initial guess for volume was
$1.05 \sum_{i} n_{i} b i$

\subsection{Parameter estimation and experimental data}

For the estimation of cavity volumes and dispersion parameters, experimental data were considered. These data consisted in pure compound saturation pressures $\left(P^{\text {sat }}\right)$ and saturated liquid molar volume $\left(v^{l}\right)$, collected from literature with the aid of NIST ThermoData Engine software [31]. The following objective function was optimized by a Nelder-Mead method [32]:

$F_{\text {obj }}=\frac{1}{\mathrm{NP}} \sum_{k=1}^{\mathrm{NP}} w\left(\frac{P_{k}^{\mathrm{sat}}-P_{k}^{\text {sat,calc }}}{P_{k}^{\mathrm{sat}}}\right)^{2}+\left(\frac{v_{k}^{l}-v_{k}^{l, \text { calc }}}{v_{k}^{l}}\right)^{2}$

where NP is the number of experimental points; 'calc' superscript denotes the quantity calculated with COSMO-SAC-Phi; and $w=10$ is a weighting factor [33-35], so that priority is given to saturation pressure data.

For saturation pressure calculations, a classical $\phi_{i}^{l}=\phi_{i}^{v}$ bubble point algorithm was used and fugacity coefficients were computed as previously described. After the bubble pressure algorithm converged, the saturated liquid volume was evaluated. To avoid the usual difficulties around the critical temperature $T_{c}$ or at very low temperatures, only data in the interval $\left[0.5 T_{c}, 0.95 T_{c}\right]$ spaced by $10 \mathrm{~K}$ were considered during the parameter optimization. 


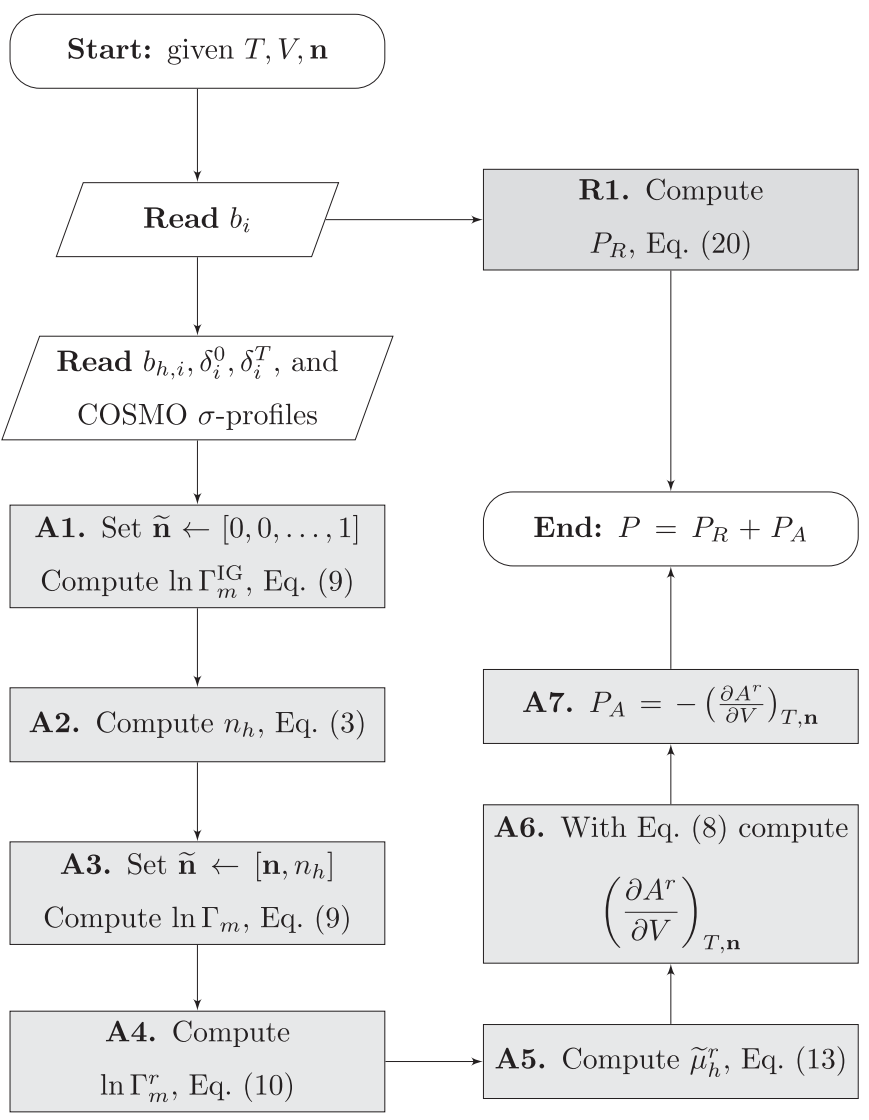

Fig. 4. Flowchart for pressure computation using the COSMO-SAC-Phi method proposed in this work, for given $T, V, \mathbf{n}$.

\section{Results and discussion}

\subsection{Pure compounds}

A few representative molecules were considered in this work as a proof of concept. The resulting parameters optimized with pure compound data, as described before, are listed in Table 1 along with per compound average relative deviations for pressure and molar volume:
$\mathrm{RD}_{P}=\frac{1}{\mathrm{NP}} \sum_{k=1}^{\mathrm{NP}}\left|\frac{P_{k}^{\mathrm{sat}}-P_{k}^{\text {sat,calc }}}{P_{k}^{\mathrm{sat}}}\right|$

$\mathrm{RD}_{v}=\frac{1}{\mathrm{NP}} \sum_{k=1}^{\mathrm{NP}}\left|\frac{v_{k}^{l}-v_{k}^{\mathrm{l}, \mathrm{calc}}}{v_{k}^{l}}\right|$

Comparison with classical Soave-Redlich-Kwong equation of state with Mathias-Copeman $\alpha$-function [36] (SRK-MC) are also included in Table 1. Recalling that three additional parameters are needed for the $\alpha$-function, taken from Ref. [36]. Regarding volume comparisons, SRK-MC results with the volume translation method proposed by Péneloux et al. [37] based solely on critical data [36] are used (SRK-MC+VT), since large deviations (average of $15 \%$ with values large than 30\%) were observed without volume correction.

As can be seen in Table 1, very good results were obtained with COSMO-SAC-Phi for both pressure and volume, being correlated with small errors. The estimated cavity volumes $b_{i}$ are physically consistent. Actually, these values are strongly correlated with the COSMO computed volumes, as can be seen in the linear fit of Fig. 5 (a). This linear relation could be used when no experimental data is available, enabling the prediction of this parameter from first principles. Further, since the estimated cavity volumes are physically consistent and Eq. (2) is always respected, holes (void) fractions in liquid and vapor phases should also be consistent. For instance, at $T=0.7 \cdot T_{C}$ for all substances tested the holes volume fraction varied between 15 and $25 \%$ in liquid phase, while the holes volume fraction is superior to $99 \%$ in vapor.

Dispersion parameters $\delta_{m}^{0}$ varied between 0.32 and $1.7 \mathrm{kcal} / \mathrm{mol}$ (per contact, allowing the direct comparison between molecules of different sizes). Smaller values were observed for light gases and hydrocarbons, and larger values were observed for alcohols and water. The same tendency was verified for the temperature dependency $\delta_{m}^{T}$ parameter. Comparing the estimated values with results of Amovilli and Mennucci [38] (based on the theory of intermolecular forces) qualitative agreement was observed. It is worth noting that results from other theories need to be divided by the molecular surface area to be compared with the results of this work.

Regarding the hole (free volume) parameter $b_{h, i}$, for most molecules a universal value around $16 \AA^{3}$ would probably be enough. However, substances with more tightly packed molecules required smaller values, always larger than the equivalent 1 Å radius sphere

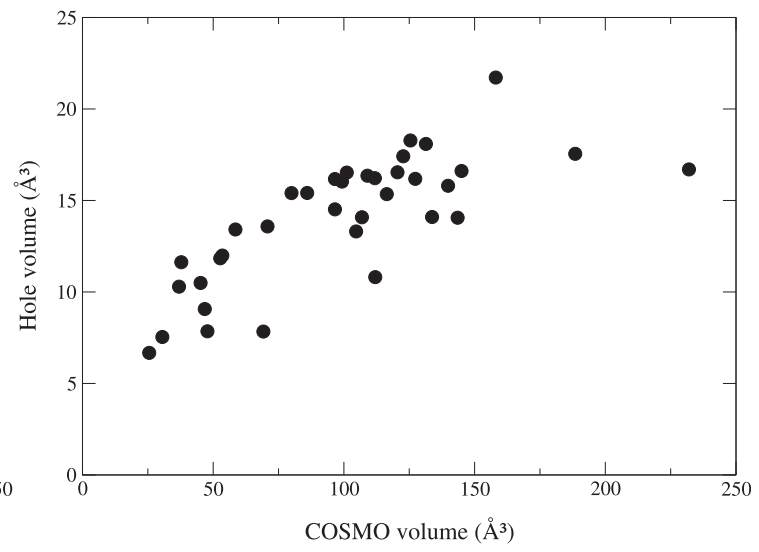

(b)

Fig. 5. Estimated cavity volumes (a) and hole volumes (b) as a function of COSMO cavity volumes computed as described in Ferrarini et al. [28]. 


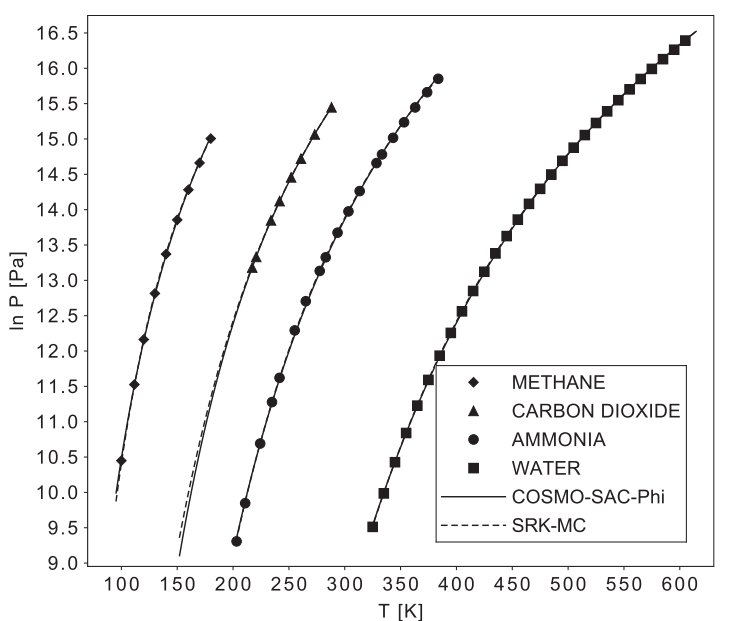

(a)

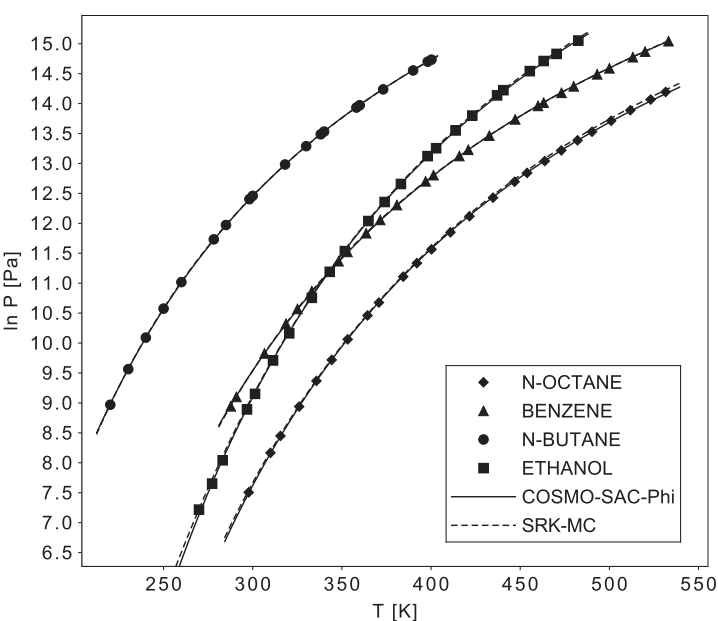

(b)

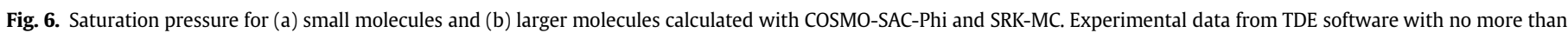
one point for every $10 \mathrm{~K}$.

$\left(4.19 \AA^{3}\right)$. As can be seen in Fig. 5 (b), there is only a weak correlation between COSMO volumes and the estimated hole (free volume) parameters $b_{h, i}$. Attempts to predict this parameter from first principles should probably also take molecular shape into account.

In Fig. 6, responses for pure compound saturation pressures of some representative molecules are shown. Even though the MCparameters used were adjusted using data from a different database software [36], for all cases the responses with COSMO-SAC-Phi and SRK-MC are visually indistinguishable and in very good agreement with experimental data.

In Fig. 7, results for saturated liquid volume of some representative molecules are shown. As can be seen, the proposed method can correlate data very well for a variety of substances, in spite of the priority for correlation of vapor pressures in the objective function Eq. (24). Some difficulties are observed with $n$-octane, probably due to the simple hard sphere model selected for the repulsive contribution. When compared with SRK-MC results, much better agreement with experimental data is observed with the proposed model. The poor results for the prediction of liquid volumes is well known for cubic equations of state. In Fig. 7 results with the volume translation method proposed by Péneloux et al. [37] are also shown, referred as SRK-MC+VT. In this work, the version based solely on critical data was considered [36]. This method indeed improved the results for cubic equation of state, but the proposed method correlation was still superior.

\subsection{VLE predictions}

In this section some vapor-liquid equilibrium (VLE) experimental data are compared with COSMO-SAC-Phi, SRK-MC+vdW, and PSRK predictions. SRK-MC+vdW corresponds to SRK equation of state with Mathias Copeman $\alpha$-function and the classical van der Waals mixing rule with no binary interaction parameters. PSRK also includes the $\mathrm{MC} \alpha$-function but relies on group-contribution binary

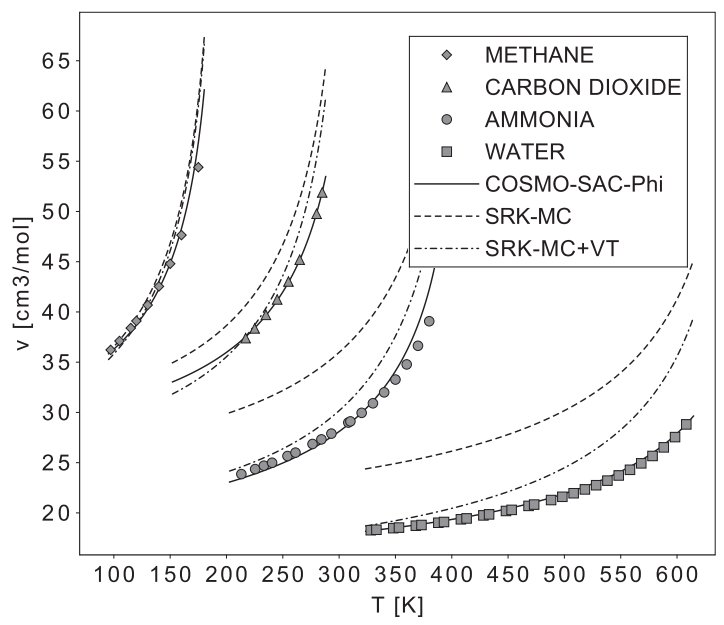

(a)

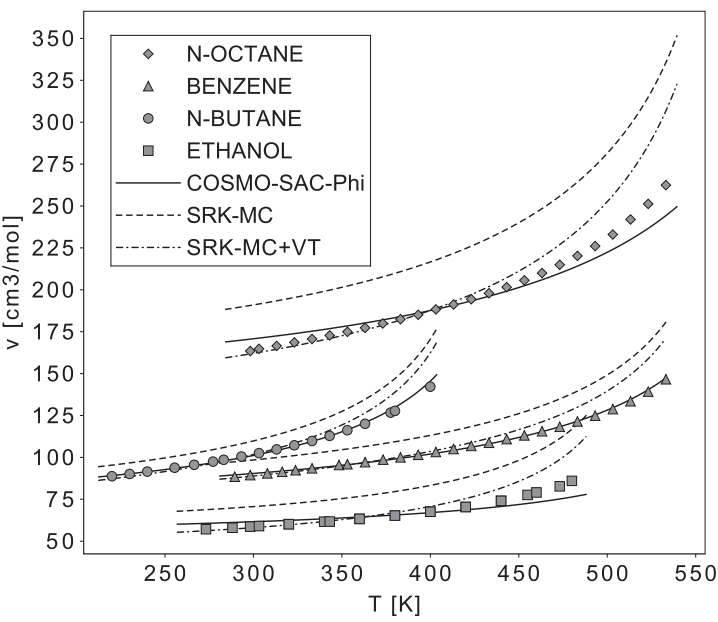

(b)

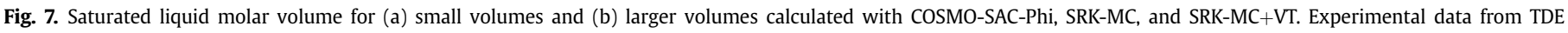
software with no more than one point for every $10 \mathrm{~K}$. 
Table 2

Relative deviations on bubble pressures for COSMO-SAC-Phi (CSP), COSMO-SAC, SRK-MC, and PSRK for different systems.

\begin{tabular}{|c|c|c|c|c|c|c|}
\hline \multirow[t]{2}{*}{ System } & \multirow[t]{2}{*}{ Ref. } & \multirow[t]{2}{*}{$P($ bar $)$} & \multicolumn{4}{|l|}{$\mathrm{RD}_{P} \%$} \\
\hline & & & CSP & COSMO-SAC & SRK-MC & PSRK \\
\hline methyl acetate/1-hexene & [39] & $0.6-1.0$ & 2.29 & 2.26 & 10.1 & 1.10 \\
\hline methanol/water & {$[40]$} & $0.2-0.8$ & 3.17 & 5.77 & 37.2 & 0.96 \\
\hline acetone/cyclohexane & [41] & $0.1-0.4$ & 5.76 & 5.59 & 27.0 & 4.63 \\
\hline chloroform/benzene & [42] & $0.3-0.7$ & 1.55 & 2.83 & 4.79 & 0.70 \\
\hline chloroform/diethyl ether & [43] & $0.2-0.7$ & 3.43 & 1.43 & 21.5 & 7.26 \\
\hline \multirow[t]{2}{*}{ chloroform/acetone } & [44] & $0.2-0.4$ & 1.02 & 1.32 & 16.3 & 1.04 \\
\hline & \multicolumn{2}{|c|}{ low pressure average } & 2.87 & 3.2 & 19.5 & 2.62 \\
\hline carbon dioxide/acetone & {$[45]$} & $5-55$ & 3.74 & - & 5.27 & 12.2 \\
\hline carbon dioxide/benzene & [46] & $15-65$ & 23.6 & - & 29.6 & 8.2 \\
\hline carbon dioxide/water & [47] & $25-150$ & 41.4 & - & $\mathrm{NC}^{\mathrm{b}}$ & 5.52 \\
\hline ammonia/n-butane & [48] & $5-25$ & 7.99 & - & 26.6 & 18.6 \\
\hline ammonia/benzene & [49] & $1-4.5$ & 9.36 & - & 41.2 & 12.6 \\
\hline ammonia/water & [50] & $1-100$ & 16.9 & - & 181 & 5.16 \\
\hline nitrogen/methane & [51] & $3-35$ & 3.46 & - & 5.83 & 1.52 \\
\hline methane/carbon dioxide & [52] & $10-80$ & 9.95 & - & 20.6 & 3.96 \\
\hline nitrogen/carbon dioxide & [53] & $5-120$ & 8.65 & - & 8.62 & 6.57 \\
\hline ethane/n-butane & [54] & $12-38$ & 4.25 & - & 2.33 & 3.08 \\
\hline ethane/n-pentane & [55] & $1-50$ & 8.54 & - & 4.59 & 8.36 \\
\hline \multirow[t]{2}{*}{ ethane $/ n$-decane } & [56] & $5-100$ & 30.1 & - & 2.86 & 33.9 \\
\hline & & overall & 10.28 & - & 26.2 & 7.52 \\
\hline
\end{tabular}

a Modified Raoult's law is assumed, high pressure systems are excluded.

b Bubble pressure not converged, see Fig. 10 (c).

interaction parameters (UNIFAC-like) combined with SRK-MC by means of a specific mixing rule [36]. Results were produced based on the iso-fugacity criteria $y_{i} \widehat{\phi}_{i}^{v}=x_{i} \widehat{\phi}_{i}$. For low pressure cases, predictions with the underlying COSMO-SAC model (GMHB1808 parametrization, as described before) were also included based on the modified Raoult's law.

Results are listed in Table 2, including references for the experimental data. Only deviations on pressure are included, since not all experiments provide vapor phase composition data. Further, deviations on pressure are usually correlated with deviations on vapor phase composition.

The proposed method provides similar responses for low pressures systems when compared with its underlying COSMO-SAC model. When compared with SRK-MC better results are obtained for almost all systems studied. The only exception is for asymmetric hydrocarbon-hydrocarbon systems, when the performance of the proposed method as well as PSRK degrades. Overall, COSMO-SACPhi performed very similarly to PSRK, but without relying on pairwise interaction parameters.
Visual comparisons are available in Figs. 8-13. In Fig. 8, some low pressure predictions are compared with experimental data for cases of positive deviations from the Raoult's law. As can be seen, COSMO-SAC-Phi responses are very similar to the ones produced with its underlying COSMO-SAC model. For all cases in this figure, COSMO-SAC based predictions are clearly superior to SRK$\mathrm{MC}+\mathrm{vdW}$ ones. The proposed method produces results very similar to PSRK for these cases.

Predictions for some low pressure cases with negative deviations from the Raoult's law are shown in Fig. 9. Again, COSMOSAC-Phi responses are very similar to the ones produced with its underlying COSMO-SAC model. For all cases in this figure, SRK$\mathrm{MC}+\mathrm{vdW}$ predicted nearly ideal behavior. This is a well-known deficiency of cubic equations of state with the classic mixing rule, unable to produce negative deviations without negative binary interaction parameters [27]. COSMO-SAC models, on the other hand, produced very good predictions for these negative deviation cases. Results with the PSRK method are also in good agreement with the experimental data.

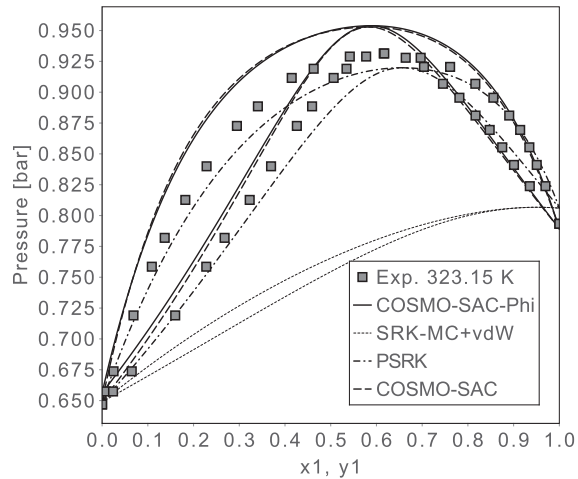

(a) methyl acetate/1-hexene

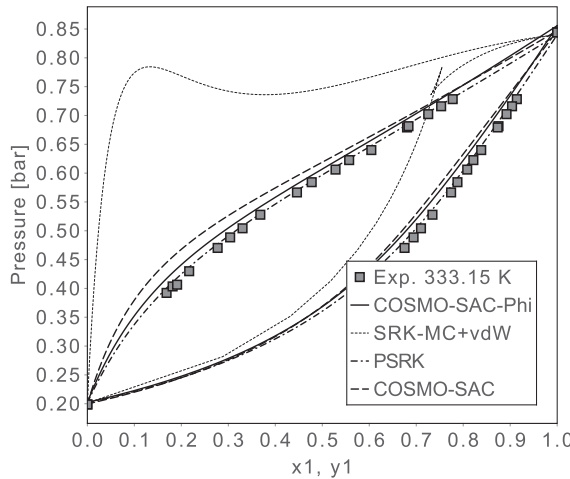

(b) methanol/water

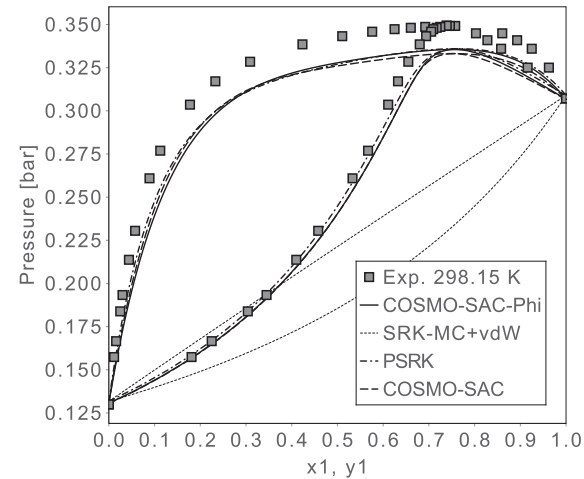

(c) acetone/cyclohexane

Fig. 8. VLE predictions for COSMO-SAC-Phi, SRK-MC+vdW, PSRK, and COSMO-SAC for low pressure cases with positive deviations from the Raoult's law. 


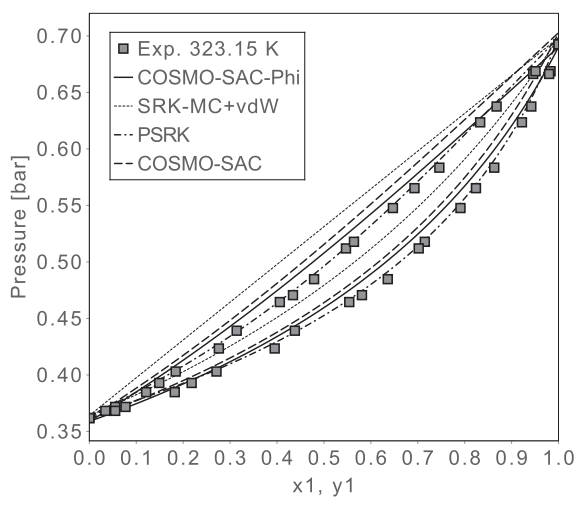

(a) chloroform/benzene

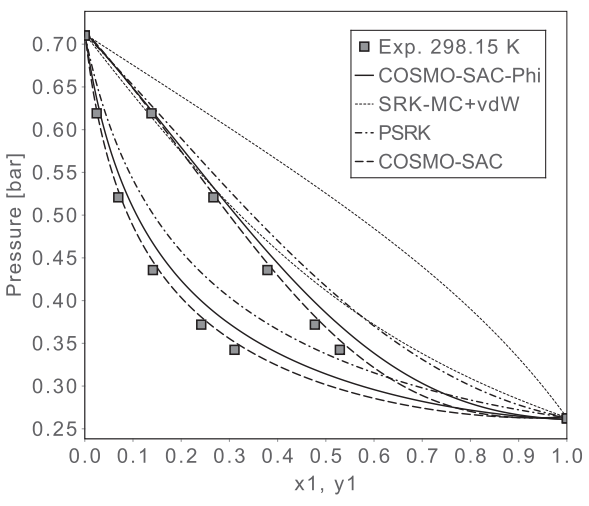

(b) chloroform/diethyl ether

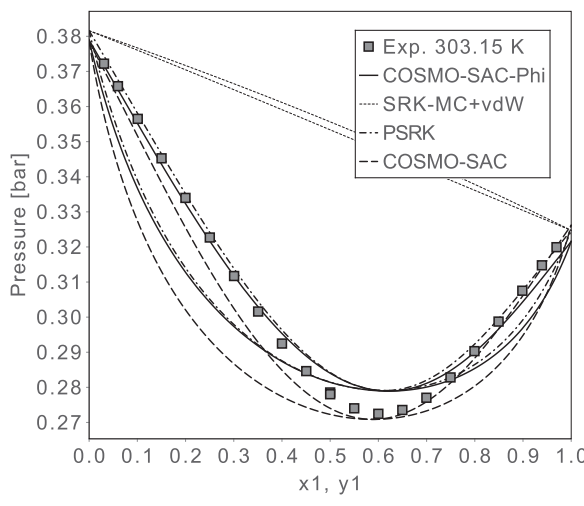

(c) chloroform/acetone

Fig. 9. VLE predictions for COSMO-SAC-Phi, SRK-MC+vdW, PSRK, and COSMO-SAC for low pressure cases with negative deviations from the Raoult's law.

Predictions for systems with carbon dioxide are shown in Fig. 10. For carbon dioxide/acetone, COSMO-SAC-Phi and SRK-MC+vdW presented similar responses, in agreement with experimental data. PSRK performance was slightly worse for this case. For mixtures with benzene and water, better qualitative agreement with experimental data is observed for the proposed model when compared with SRK-MC+vdW. Both positive and negative deviations are correctly predicted for these last two cases, including an improved quantitative prediction for the diluted region for carbon dioxide/water. For these last two cases, PSRK produced much better results, but we should note that the method contains specific binary parameters for these molecules.

Predictions for systems with ammonia are shown in Fig. 11. Again SRK-MC+vdW without binary interaction parameters could not predict the strong deviations experimentally observed. COSMO-SAC-Phi, on the other hand, produced very good predictions for positive deviations as well as negative, with pressures up to 100 bar. PSRK was superior to the proposed method only for the ammonia/water pair.

In Fig. 12, VLE calculations for some light gas mixtures are shown. In these cases, good agreement with experimental data was observed for all models, with a slightly better performance for PSRK followed by COSMO-SAC-Phi.

In Fig. 13, ethane/hydrocarbon mixtures are used to verify how the proposed model handles asymmetric systems. As can be seen, as the system asymmetry becomes higher, the proposed model as well as PSRK deviate more from experimental data. This could be probably improved in future works by a more sophisticated repulsion term or a better mixing rule for the hole (free volume) parameter. For these cases SRK-MC+vdW showed better agreement with experimental data.

\subsection{LLE predictions}

In this section some liquid-liquid equilibrium (LLE) experimental data are compared with the proposed model, its underlying COSMO-SAC, and UNIFAC-LLE [57]. For COSMO-SAC-Phi calculations, the iso-fugacity criteria $x_{i}^{\alpha} \widehat{\phi}_{i}^{\alpha}=x_{i}^{\beta} \widehat{\phi}_{i}^{\beta}$ was considered, where $\alpha$ and $\beta$ are the two liquid phases in equilibrium. Additionally, as the temperature rises, pressure is also increased to assure no vapor phase appears. For the UNIFAC-LLE model as well as COSMO-SAC alone, the iso-activity $x_{i}^{\alpha} \gamma_{i}^{\alpha}=x_{i}^{\beta} \gamma_{i}^{\beta}$ criteria was considered and pressure was disregarded.

Numerical deviations for all cases tested are listed in Table 3, considering the average absolute deviation in molar fraction of the first compound in both phases:

$\mathrm{AD}_{x_{1}}=\frac{1}{2 \mathrm{NP}} \sum_{k=1}^{\mathrm{NP}}\left|x_{1, k}^{\alpha}-x_{1, k}^{\alpha, \text { calc }}\right|+\left|x_{1, k}^{\beta}-x_{1, k}^{\beta, \text { calc }}\right|$

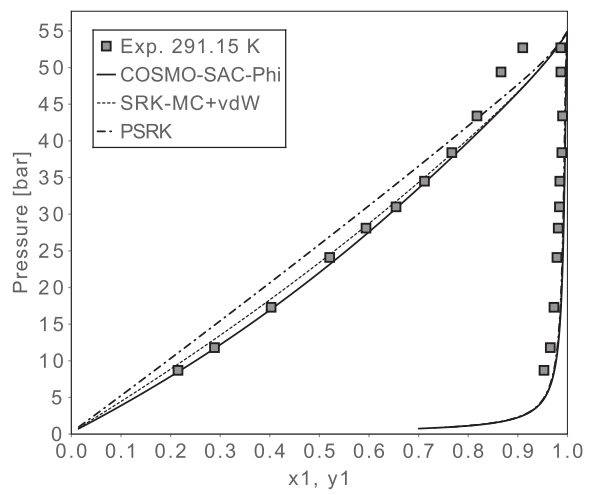

(a) carbon dioxide/acetone

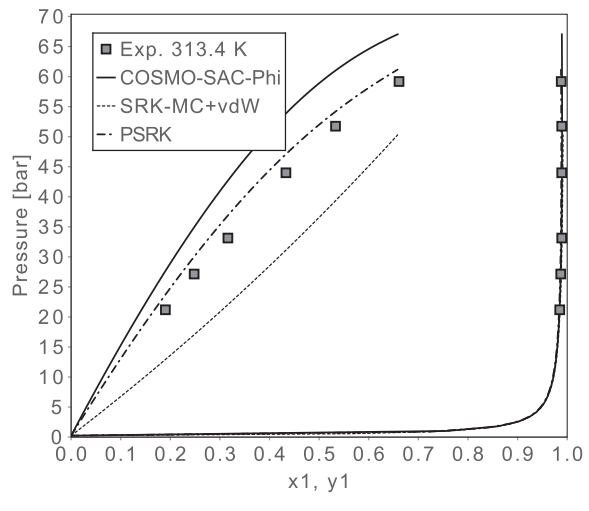

(b) carbon dioxide/benzene

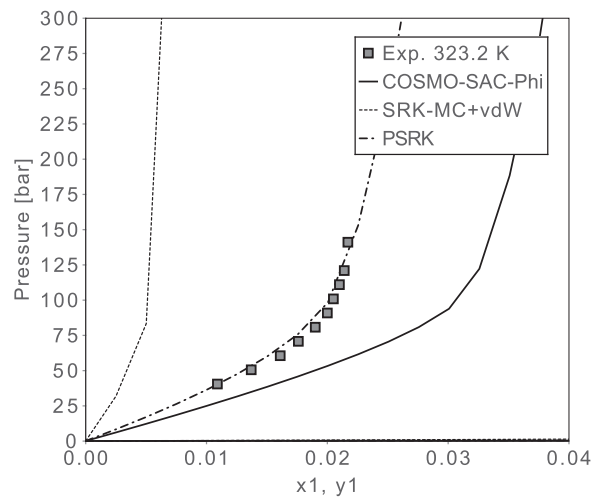

(c) carbon dioxide/water

Fig. 10. VLE predictions for COSMO-SAC-Phi, SRK-MC+vdW, and PSRK for carbon dioxide systems. 


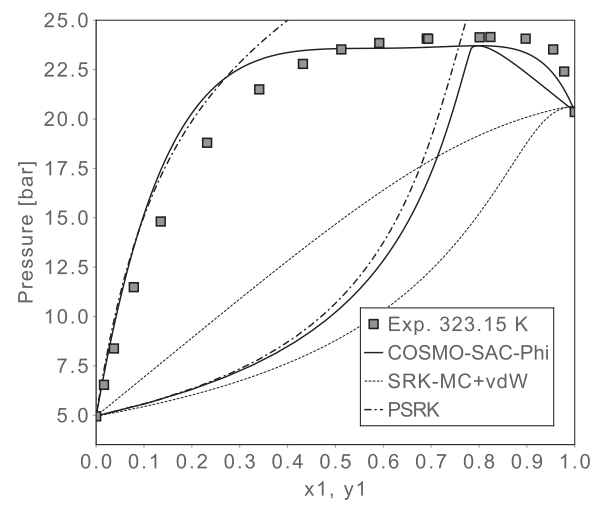

(a) ammonia/n-butane

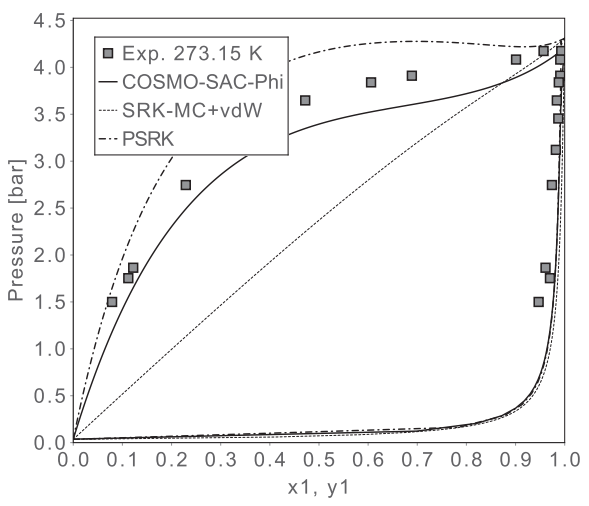

(b) ammonia/benzene

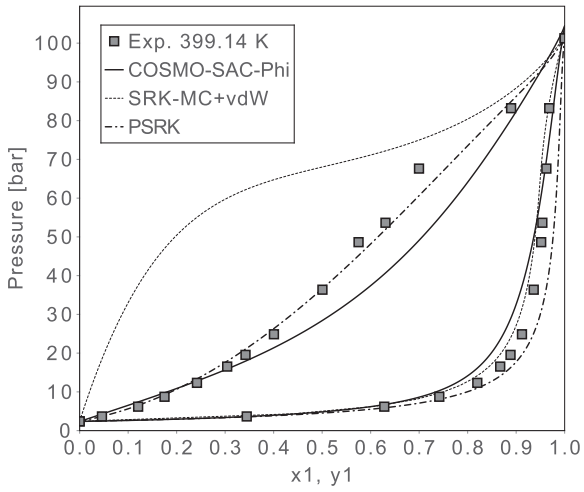

(c) ammonia/water

Fig. 11. VLE predictions for COSMO-SAC-Phi, SRK-MC+vdW, and PSRK for ammonia systems.

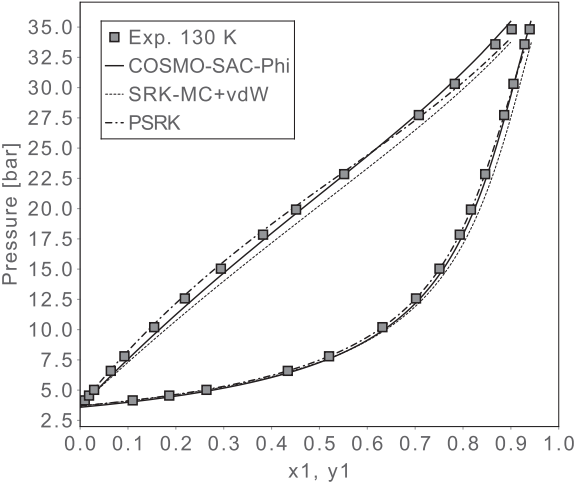

(a) nitrogen/methane

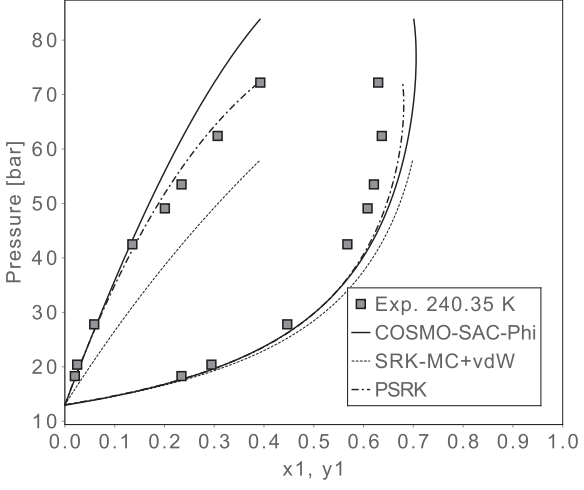

(b) methane/carbon dioxide

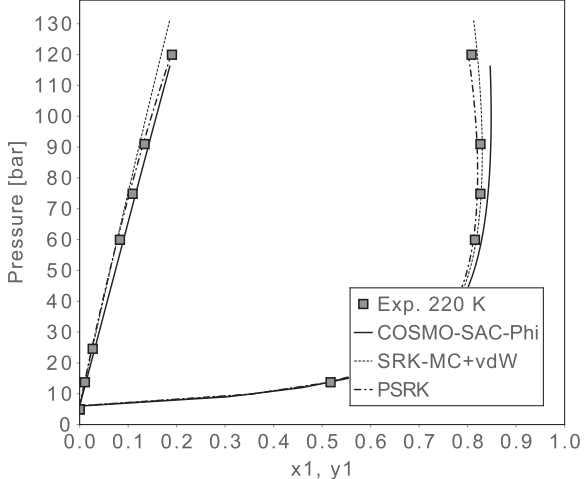

(c) nitrogen/carbon dioxide

Fig. 12. VLE predictions for COSMO-SAC-Phi, SRK-MC+vdW, and PSRK for light gas systems.

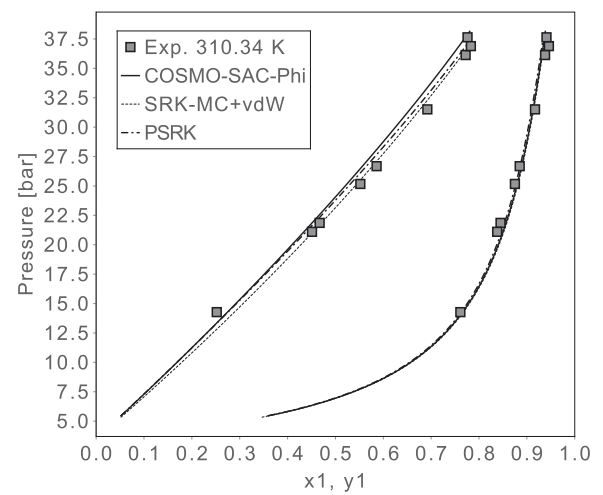

(a) ethane $/ n$-butane

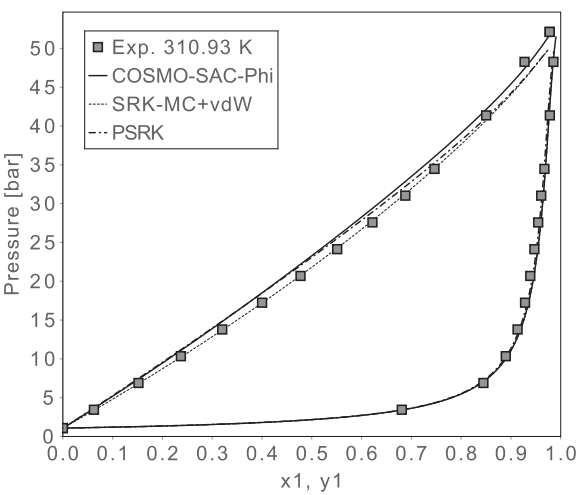

(b) ethane $/ n$-pentane

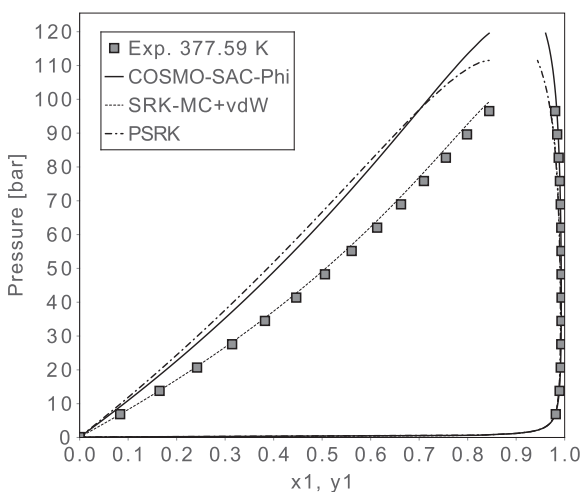

(c) ethane $/ n$-decane

Fig. 13. VLE predictions for COSMO-SAC-Phi, SRK-MC+vdW, and PSRK for ethane systems with different degrees of asymmetry.

In Fig. 14 different binary mutual solubilities are predicted. The proposed model was able to predict different scenarios, from highly soluble ( $n$-pentanol/water) to nearly immiscible systems (hydrocarbon/water at low temperature). Further, the solubility increase with the temperature (and pressure) was correctly reproduced. At low temperatures (and pressures) COSMO-SAC-Phi and its underlying COSMO-SAC model produce very similar responses. Naturally, COSMO-SAC alone is unable to correctly predict the increased mutual solubilities due to pressure. At low temperatures, COSMO-SAC models under-predicted water solubility in $n$-pentanol rich phase, Fig. 14 (a). It is also noteworthy that for theses cases the UNIFAC-LLE responses are actually extrapolations, since the 
Table 3

Absolute deviations for the molar fraction of the first compound in LLE cases for COSMO-SAC-Phi, COSMO-SAC, and UNIFAC-LLE for different systems.

\begin{tabular}{|c|c|c|c|c|}
\hline \multirow[t]{2}{*}{ System } & \multirow[t]{2}{*}{ Ref. } & \multicolumn{3}{|l|}{$\mathrm{AD}_{x_{1}}$} \\
\hline & & COSMO-SAC-Phi & COSMO-SAC & UNIFAC-LLE \\
\hline n-pentanol/water & [58] & 0.037 & 0.044 & 0.042 \\
\hline 1-hexene/water & [59] & 0.001 & 0.001 & 0.001 \\
\hline n-octane/water & [60] & 0.001 & 0.002 & 0.004 \\
\hline methanol/benzene/water & [61] & 0.010 & 0.010 & 0.019 \\
\hline n-butanol/cyclohexane/water & [61] & 0.030 & 0.031 & 0.005 \\
\hline
\end{tabular}

method was developed for temperatures between 10 and $40{ }^{\circ} \mathrm{C}$.

Results for some ternary systems are shown in Fig. 15. For the cases studied, good predictions were obtained with COSMO-SACPhi. Once more water solubility in alcohol was underestimated (as in Fig. 14), but the tie-lines are in excellent agreement with experimental data. Results with COSMO-SAC alone, are almost indistinguishable from COSMO-SAC-Phi in these cases (see Table 3) and were not included in this figure. This is because at room temperature (and low pressure) the proposed modifications are of minor importance in the liquid phase.

\subsection{Effects on activity coefficients}

When compared with COSMO-SAC, the proposed model adds dispersion and free volume. As already observed in VLE and LLE predictions, the effect of the proposed extension is small on activity coefficients at low pressures. In this section the effects are further investigated by directly comparing activity coefficients computed with COSMO-SAC alone and by means of the proposed method. Activity coefficients can be computed by the following expression when using equations of state:

$\ln \gamma_{i}=\ln \widehat{\phi}_{i}-\ln \phi_{i}$

where $\widehat{\phi}_{i}$ and $\phi_{i}$ are the fugacity coefficients of compound $i$ in mixture and pure, respectively, on the same conditions of temperature and pressure (selecting the liquid-like root).

In Fig. 16 activity coefficients for different mixtures are compared. Overall COSMO-SAC-Phi responses are very similar to the COSMO-SAC ones, with a maximum difference of $20 \%$ for the activity coefficients in logarithmic units. The discrepancies are small mainly because the additional effects partially cancel out in excess properties or activity coefficients (see Eq. (28)).
In Fig. 16 (a) compounds with similar free volume and dispersion parameters are considered. For this case the difference between the models is minimal. For the case in Fig. 16 (b), similar dispersion parameters are considered but with diverse free volume parameters. Finally, in Fig. 16 (c) compounds have similar free volume parameter but very different dispersion parameters. This is the case with more pronounced difference between the models.

\section{Conclusions}

In this work a new family of equations of state is proposed, directly combining COSMO-SAC and lattice-fluid ideas. The proposed model, called COSMO-SAC-Phi or CSP for short, can take into account pressure-volume effects by the addition of holes representing free volume. This characteristic is not present in COSMO-RS, COSMO-SAC, or F-SAC models.

The proposed model can also be seen as a perturbation model. In the proof of concept present in this work, a simple hard sphere model was considered for the repulsion forces (reference fluid), corrected with a COSMO-based perturbation. Pure compound parameters were introduced accounting for dispersive interactions. The model was able to correlate very well experimental saturation pressure and liquid volume simultaneously, with average deviations of $1.16 \%$ and $1.59 \%$, respectively.

Predictions of mixture vapor-liquid equilibrium (VLE) data with COSMO-SAC-Phi performed similarly to PSRK and better than SRKMC for most cases. Mixtures ranging from light gases to molecules with up 10 carbons were tested for low pressures as well as for pressures over 100 bar. Binary and ternary liquid-liquid equilibrium (LLE) predictions were also in good agreement with experimental data for systems including alcohol/hydrocarbons/water. For the LLE cases the performance was similar to the observed with UNIFACLLE, with improved responses at high temperatures and pressures.

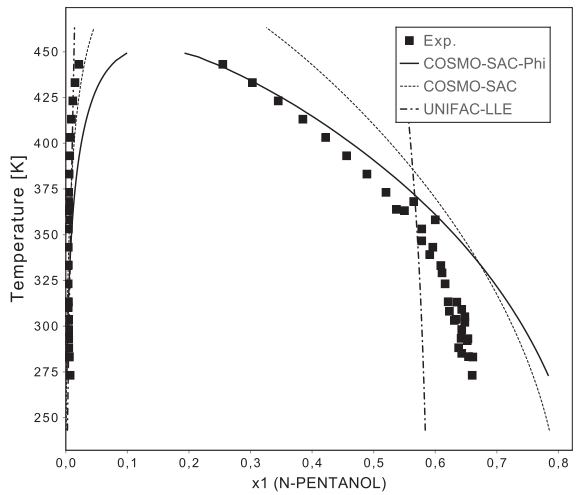

(a) $n$-pentanol/water [58]

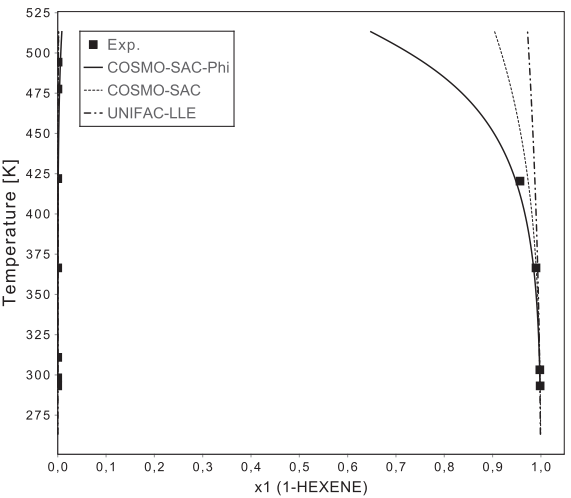

(b) 1-hexene/water [59]

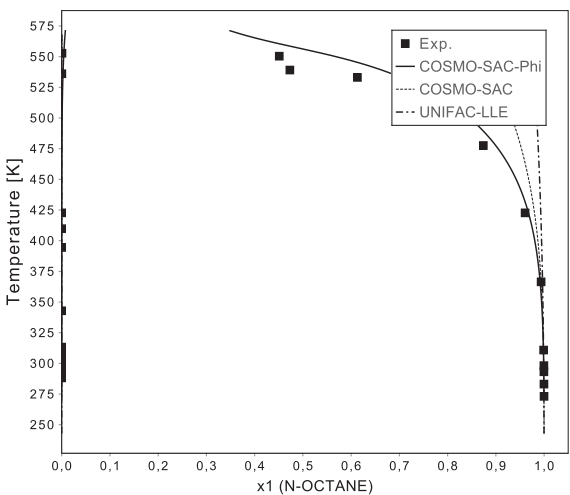

(c) $n$-octane/water $[60]$

Fig. 14. LLE predictions using COSMO-SAC-Phi and UNIFAC-LLE models for aqueous systems with different degrees of solubility. 


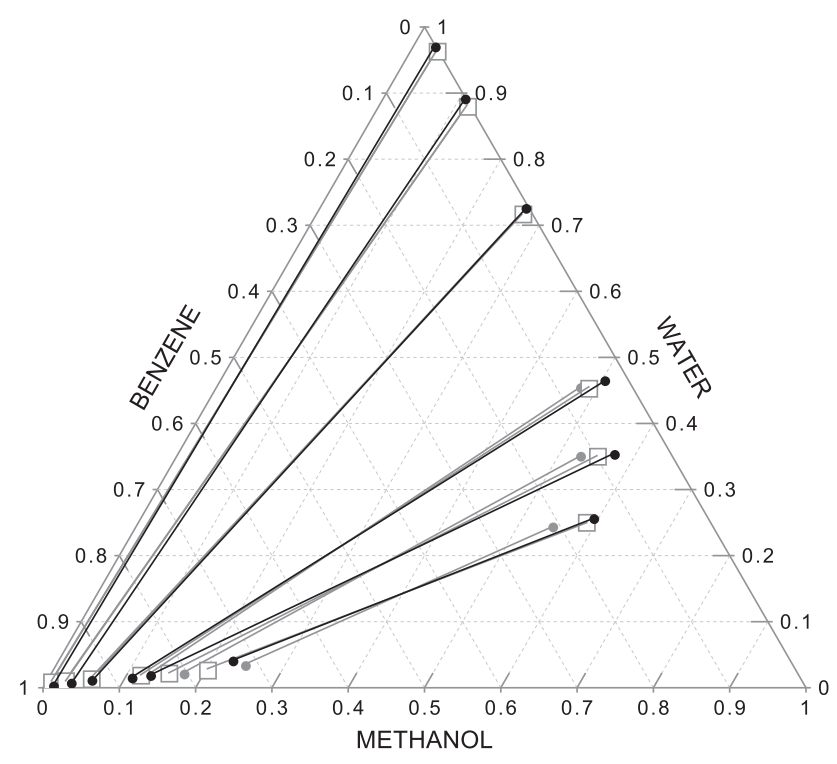

- Exp. Data, 303.2 K $\square$ COSMO-SAC-Phi • UNIFAC-LLE

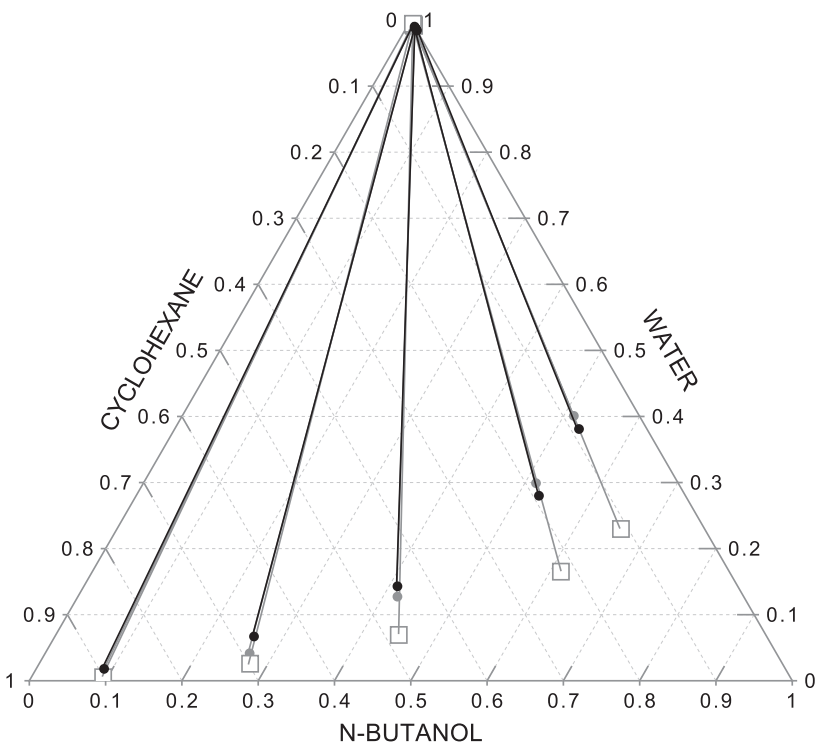

- Exp. Data, 298.15 K $\square$ COSMO-SAC-Phi - UNIFAC-LLE

(a) methanol/benzene/water [61]

(b) $n$-butanol/cyclohexane/water [61]

Fig. 15. LLE predictions for COSMO-SAC-Phi and UNIFAC-LLE for ternary systems.

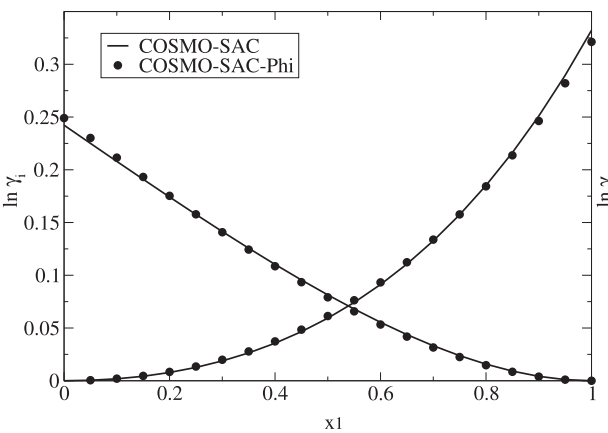

(a) n-butane/diethyl ether

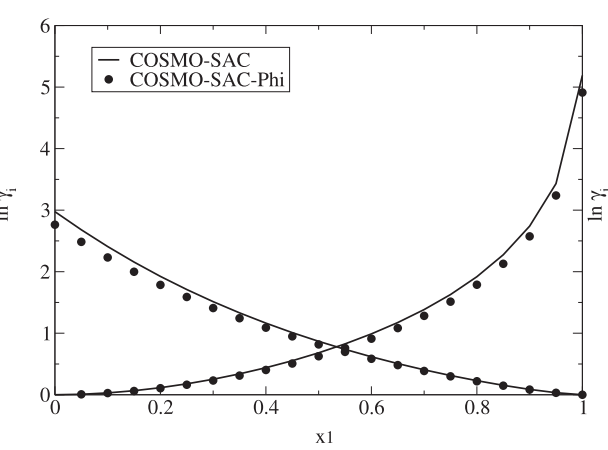

(b) n-butane/methanol

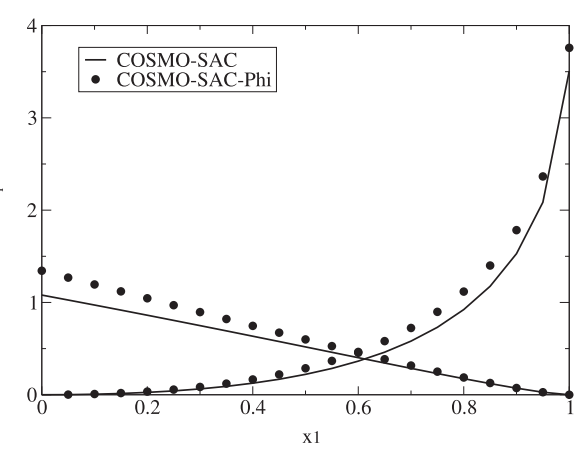

(c) $n$-butane $/ n$-pentanol

Fig. 16. Comparison of activity coefficients computed with COSMO-SAC and COSMO-SAC-Phi at $273 \mathrm{~K}$ and $1 \mathrm{~atm}$.

Future works can improve the repulsive contribution as well as the description of free volume. The development of a fully predictive version, without the need of compound specific volume and dispersion parameters should also be pursued.

\section{Acknowledgement}

This work was partially supported by CNPq-Brazil under grant no. 304046/2016-7 and by CAPES-Brazil.

\section{References}

[1] Andreas Klamt, Conductor-like screening model for real solvents: a new approach to the quantitative calculation of solvation phenomena, J. Phys. Chem. 99 (7) (feb 1995) 2224-2235, https://doi.org/10.1021/j100007a062.

[2] Shiang-Tai Lin, Stanley I. Sandler, A priori phase equilibrium prediction from a segment contribution solvation model, Ind. Eng. Chem. Res. 41 (5) (mar 2002) 899-913, https://doi.org/10.1021/ie001047w.

[3] Rafael De P. Soares, Renan P. Gerber, Functional-segment activity coefficient model. 1. Model formulation, Ind. Eng. Chem. Res. 52 (32) (aug 2013) 11159-11171, https://doi.org/10.1021/ie400170a.

[4] Rafael de P. Soares, Renan P. Gerber, Luiz F.K. Possani, Paula B. Staudt, Functional-segment activity coefficient model. 2. Associating mixtures, Ind. Eng. Chem. Res. 52 (32) (jul 2013) 11172-11181, https://doi.org/10.1021/ ie4013979.

[5] Costas Panayiotou, Equation-of-State models and quantum mechanics calculations, Ind. Eng. Chem. Res. 42 (7) (apr 2003) 1495-1507, https://doi.org/ 10.1021/ie0207212.

[6] Costas Panayiotou, Solvation thermodynamics and non-randomness. Part I: self-solvation, J. Chem. Eng. Data 55 (12) (2010) 5453-5464, https://doi.org/ 10.1021/je100575q.

[7] C. Panayiotou, I. Tsivintzelis, D. Aslanidou, V. Hatzimanikatis, Solvation quantities from a COSMO-RS equation of state, J. Chem. Thermodyn. 90 (2015) 294-309, https://doi.org/10.1016/j.jct.2015.07.011.

[8] Yusuke Shimoyama, Yoshio Iwai, Development of activity coefficient model based on COSMO method for prediction of solubilities of solid solutes in supercritical carbon dioxide, J. Supercrit. Fluids 50 (3) (2009) 210-217, https:// doi.org/10.1016/j.supflu.2009.06.004.

[9] Cauê T.O.G. Costa, Frederico W. Tavares, Argimiro R. Secchi, Equation of state based on the hole-lattice theory and surface-charge density (COSMO): Part A pure compounds, Fluid Phase Equilib. 409 (feb 2016) 472-481, https:/| 
doi.org/10.1016/j.fluid.2015.11.010.

[10] Cauê T.O.G. Costa, Frederico W. Tavares, Argimiro R. Secchi, Equation of state based on the hole-lattice theory and surface-charge density (COSMO): Part B vapor-liquid equilibrium for mixtures, Fluid Phase Equilib. 419 (1-10) (jul 2016), https://doi.org/10.1016/j.fluid.2016.03.005.

[11] Silvana Mattedi, Frederico W. Tavares, Marcelo Castier, Group contribution equation of state based on the lattice fluid theory: alkane-alkanol systems, Fluid Phase Equilib. 142 (1-2) (jan 1998), https://doi.org/10.1016/S03783812(97)00218-5, 33-54

[12] Ming-Tsung Lee, Shiang-Tai Lin, Prediction of mixture vapor-liquid equilibrium from the combined use of Peng-Robinson equation of state and COSMOSAC activity coefficient model through the Wong-Sandler mixing rule, Fluid Phase Equilib. 254 (1-2) (jun 2007) 28-34, https://doi.org/10.1016 j.fluid.2007.02.012.

[13] ChiehMing Hsieh, ShiangTai Lin, Determination of cubic equation of state parameters for pure fluids from first principle solvation calculations, AIChE J. 54 (8) (aug 2008) 2174-2181, https://doi.org/10.1002/aic.11552.

[14] A. Ben-Naim, Solvation Thermodynamics, Plenum Press, 1987. ISBN 1475765525.

[15] Chieh-Ming Hsieh, Shiang-Tai Lin, Prediction of 1-octanol-water partition coefficient and infinite dilution activity coefficient in water from the PR+COSMOSAC model, Fluid Phase Equilib. 285 (1-2) (nov 2009) 8-14 https://doi.org/10.1016/j.fluid.2009.06.009.

[16] Chieh-Ming Hsieh, Shiang-Tai Lin, First-principles predictions of vapor-liquid equilibria for pure and mixture fluids from the combined use of cubic equations of state and solvation calculations, Ind. Eng. Chem. Res. 48 (6) (mar 2009) 3197-3205, https://doi.org/10.1021/ie801118a.

[17] Paula B. Staudt, Rafael D.P. Soares, A self-consistent Gibbs excess mixing rule for cubic equations of state, Fluid Phase Equilib. 334 (nov 2012) 76-88, https://doi.org/10.1016/j.fluid.2012.06.029.

[18] Li-Hsin Wang, Chieh-Ming Hsieh, Shiang-Tai Lin, Prediction of gas and liquid solubility in organic polymers based on the PR+COSMOSAC equation of state, Ind. Eng. Chem. Res. 57 (31) (jun 2018) 10628-10639, https://doi.org/ 10.1021/acs.iecr.8b01780.

[19] David Shan Hill Wong, Stanley I. Sandler, A theoretically correct mixing rule for cubic equations of state, AIChE J. 38 (5) (may 1992) 671-680, https:// doi.org/10.1002/aic.690380505.

[20] Michael L. Michelsen, A modified Huron-Vidal mixing rule for cubic equations of state, Fluid Phase Equilib. 60 (1-2) (oct 1990) 213-219, https://doi.org/ 10.1016/0378-3812(90)85053-D.

[21] B.E. Poling, J.M. Prausnitz, J.P. O'Connell, The Properties of Gases and Liquids 5E. McGraw Hill Professional, McGraw-Hill Education, 2000. ISBN 9780071499996.

[22] I.C. Sanchez, R.H. Lacombe, An elementary molecular theory of classical fluids. pure fluids, J. Phys. Chem. 80 (21) (1976) 2352-2362.

[23] Chongli Zhong, Wenchuan Wang, Huanzhang Lu, Simplified hole theory equation of state for polymer liquids, Fluid Phase Equilib. 86 (1993) 137-146, https://doi.org/10.1016/0378-3812(93)87172-W.

[24] M.L. Michelsen, J.M. Mollerup, Thermodynamic Models: Fundamentals \& Computational Aspects, Tie-Line Publications, 2007. ISBN 9788798996132.

[25] Norman F. Carnahan, Kenneth E. Starling, Equation of state for nonattracting rigid spheres, J. Chem. Phys. 51 (2) (1969) 635-636, https://doi.org/10.1063/ 1.1672048 .

[26] Amra Tihic, Georgios M. Kontogeorgis, Nicolas Von Solms, Michael L. Michelsen, A predictive group-contribution simplified PC-SAFT equation of state: application to polymer systems, Ind. Eng. Chem. Res. 47 (15) (2008) 5092-5101, https://doi.org/10.1021/ie0710768.

[27] K. Georgios, Folas Georgios M. Kontogeorgis, Thermodynamic Models for Industrial Applications: from Classical and Advanced Mixing Rules to Association Theories, John Wiley \& Sons, 2010, https://doi.org/10.1002/ 9780470747537. ISBN 9780470697269.

[28] F. Ferrarini, G.B. Flores, A.R. Muniz, R.P. Soares, An open and extensible sigmaprofile database for cosmo-based models, AIChE J. 64 (9) (2018) 3443-3455 https://doi.org/10.1002/aic.16194.

[29] R.P. Gerber, R.D.P. Soares, Assessing the reliability of predictive activity coefficient models for molecules consisting of several functional groups, Braz. J. Chem. Eng. 30 (1) (mar 2013) 1-11, https://doi.org/10.1590/S010466322013000100002

[30] G.B. Flôres, P.B. Staudt, R.D.P. Soares, Including dispersive interactions in the F-SAC model, Fluid Phase Equilib. 426 (oct 2016) 56-64, https://doi.org/ 10.1016/j.fluid.2016.02.043.

[31] Michael Frenkel, Robert D. Chirico, Vladimir Diky, Xinjian Yan, Qian Dong, Chris uzny, ThermoData engine (TDE): software implementation of the dynamic data evaluation concept, J. Chem. Inf. Model. 45 (4) (jul 2005) 816-838, https://doi.org/10.1021/ci050067b.

[32] J.A. Nelder, R. Mead, A simplex method for function minimization, Comput. J. 7 (4) (jan 1965) 308-313, https://doi.org/10.1093/comjnl/7.4.308.

[33] Jens M. Sørensen, Thomas Magnussen, Peter Rasmussen, Aage Fredenslund, Liquid-liquid equilibrium data: their retrieval, correlation and prediction Part II: Correlation, Fluid Phase Equilib. 3 (1) (1979) 47-82, https://doi.org/ 10.1016/0378-3812(79)80027-8.

[34] L.F.K. Possani, G.B. Flôres, P.B. Staudt, R.D.P. Soares, Simultaneous correlation of infinite dilution activity coefficient, vapor-liquid, and liquid-liquid equilibrium data with F-SAC, Fluid Phase Equilib. 364 (feb 2014) 31-41, https:// doi.org/10.1016/j.fluid.2013.11.040.
[35] Neumara Bender, Nilo Srgio Medeiros Cardozo, R. de P. Soares, Avoiding binary interaction parameters in the gc-pc-saft model with a parametrization based in vle and idac data: n-alkanes and 1-alkanols, Fluid Phase Equilib. 412 (2016) 9-20, https://doi.org/10.1016/j.fluid.2015.11.036.

[36] Sven Horstmann, Jabłoniec Anna, Jörg Krafczyk, Kai Fischer, Jürgen Gmehling, PSRK group contribution equation of state: comprehensive revision and extension IV, including critical constants and $\alpha$-function parameters for 1000 components, Fluid Phase Equilib. 227 (2) (jan 2005) 157-164, https://doi.org/ 10.1016/j.fluid.2004.11.002.

[37] André Péneloux, Evelyne Rauzy, Richard Fréze, A consistent correction for Redlich-Kwong-Soave volumes, Fluid Phase Equilib. 8 (1) (1982) 7-23.

[38] Claudio Amovilli, Benedetta Mennucci, Self-consistent-field calculation of pauli repulsion and dispersion contributions to the solvation free energy in the polarizable continuum model, J. Phys. Chem. B 101 (6) (1997) 1051-1057, https://doi.org/10.1021/jp9621991.

[39] Juergen Gmehling, Isothermal vapor-liquid equilibriums in binary systems formed by esters with alkenes, J. Chem. Eng. Data 28 (1) (1983) 27-30, https://doi.org/10.1021/je00031a008.

[40] Kiyofumi Kurihara, Tsuyoshi Minoura, Kouichi Takeda, Kazuo Kojima, Isothermal vapor-liquid equilibria for methanol + ethanol + water, methanol + water, and ethanol + water, J. Chem. Eng. Data 40 (3) (1995) 679-684, https://doi.org/10.1021/je00019a033.

[41] Henry V. Kehiaian, Silvia Porcedda, Bruno Marongiu, Luciano Lepori, Enrico atteoli, Thermodynamics of binary mixtures containing linear or cyclic alkanones + n-alkanes or + cycloalkanes, Fluid Phase Equilib. 63 (3) (1991) 231-257, https://doi.org/10.1016/0378-3812(91)80035-T.

[42] Isamu Nagata, Hiromichi Hayashida, Vapor-liquid equilibrium data for the ternary systems: methyl acetate-2-propanol-benzene and methyl acetatechloroform-benzene, J. Chem. Eng. Jpn. 3 (2) (1970) 161-166, https://doi.org/ 10.1252/jcej.3.161.

[43] Yash Paul Handa, David Edward Jones, Vapor pressures of triethylamine + chloroform and of diethyl ether + chloroform at 298.15k, Can. J. Chem. 53 (21) (1975) 3299-3304, https://doi.org/10.1139/v75-470.

[44] Jay A. Hopkins, Venkat R. Bhethanabotla, Scott W. Campbell, Total pressure measurements for chloroform + acetone + toluene at $303.15 \mathrm{k}$, J. Chem. Eng. Data 39 (3) (1994) 488-492, https://doi.org/10.1021/je00015a019.

[45] Chany-Yih Day, Chiehming J. Chang, Chiu-Yang Chen, Phase equilibrium of ethanol $+\mathrm{CO} 2$ and acetone $+\mathrm{CO} 2$ at elevated pressures, J. Chem. Eng. Data 41 (4) (1996) 839-843, https://doi.org/10.1021/je960049d.

[46] Choon-Ho Kim, P. Vimalchand, Marc D. Donohue, Vapor-liquid equilibria for binary mixtures of carbon dioxide with benzene, toluene and p-xylene, Fluid Phase Equilib. 31 (3) (1986) 299-311, https://doi.org/10.1016/0378-3812(86) 87014-5.

[47] A. Bamberger, G. Sieder, G. Maurer, High-pressure (vapor+liquid) equilibrium in binary mixtures of (carbon dioxide+water or acetic acid) at temperatures from 313 to 353 k, J. Supercrit. Fluids 17 (2) (2000) 97-110, https://doi.org/ 10.1016/S0896-8446(99)00054-6.

[48] Neil F. Giles, Howard L. Wilson, W. Vincent Wilding, Phase equilibrium measurements on twelve binary mixtures, J. Chem. Eng. Data 41 (6) (1996) 1223-1238, https://doi.org/10.1021/je960161b.

[49] Katsuji Noda, Tetsuo Morisue, Kiyoharu Ishida, Vapor-liquid equilibria for the benzene-cyclohexene-ammonia system, J. Chem. Eng. Jpn. 8 (2) (1975) 104-108, https://doi.org/10.1252/jcej.8.104.

[50] Syed S.H. Rizvi, Robert A. Heidemann, Vapor-liquid equilibria in the ammoniawater system, J. Chem. Eng. Data 32 (2) (1987) 183-191, https://doi.org/ $10.1021 / \mathrm{je} 00048 \mathrm{a} 017$.

[51] A.J. Kidnay, R.C. Miller, W.R. Parrish, M.J. Hiza, Liquid-vapour phase equilibria in the N2 CH4 system from 130 to $180 \mathrm{k}$, Cryogenics 15 (9) (1975) 531-540, https://doi.org/10.1016/0011-2275(75)90149-6.

[52] Qazi Nasir, Khalik M. Sabil, K.K. Lau, Measurement of isothermal (vapor+liquid) equilibria, (vle) for binary $(\mathrm{CH} 4+\mathrm{CO} 2)$ from $\mathrm{t}=(240.35$ to 293.15$) \mathrm{k}$ and $\mathrm{CO} 2$ rich synthetic natural gas systems from $\mathrm{t}=(248.15$ to 279.15$) \mathrm{k}$. J. Nat. Gas Sci. Eng. 27 (2015) 158-167, https://doi.org/10.1016/j.jngse.2015.08.045.

[53] T.S. Brown, E.D. Sloan, A.J. Kidnay, Vapor-liquid equilibria in the nitrogen + carbon dioxide + ethane system, Fluid Phase Equilib. 51 (1989) 299-313, https://doi.org/10.1016/0378-3812(89)80372-3.

[54] J.G. Dingrani, Thodos George, Vapor-liquid equilibrium behavior of the ethane-n-butane-n-hexane system, Can. J. Chem. Eng. 56 (5) (1978) 616-623, https://doi.org/10.1002/cjce.5450560516.

[55] H.H. Reamer, B.H. Sage, W.N. Lacey, Phase equilibria in hydrocarbon systems. volumetric and phase behavior of the ethane-n-pentane system, J. Chem. Eng. Data 5 (1) (1960) 44-50, https://doi.org/10.1021/je60005a012.

[56] H.H. Reamer, B.H. Sage, Phase equilibria in hydrocarbon systems. volumetric and phase behavior of the ethane-n-decane system, J. Chem. Eng. Data 7 (2) (1962) 161-168, https://doi.org/10.1021/je60013a001.

[57] Thomas Magnussen, Peter Rasmussen, Aage Fredenslund, UNIFAC parameter table for prediction of liquid-liquid equilibriums, Ind. Eng. Chem. Process Des. Dev. 20 (2) (apr 1981) 331-339, https://doi.org/10.1021/i200013a024.

[58] Andrzej Maczynski, David G. Shaw, Marian Goral, Barbara WisniewskaGoclowska, IUPAC-NIST solubility data series. 82: alcohols with water-revised and updated: Part 2. C5 alcohols with water, J. Phys. Chem. Ref. Data 36 (1) (2007) 133, https://doi.org/10.1063/1.2366719.

[59] Marian Goral, Barbara Wisniewska-Goclowska, Skrzecz Adam, Iwona Owczarek, Krystyna Blazej, Marie-Claire Haulait-Pirson, Glenn T. Hefter, Zofia Maczynska, Andrzej Szafranski, Colin L. Young, Iupac-nist 
solubility data series. 81 . hydrocarbons with water and seawater-revised and updated. part 3. $\mathrm{C} 6 \mathrm{H} 8-\mathrm{C} 6 \mathrm{H} 12$ hydrocarbons with water and heavy water, J. Phys. Chem. Ref. Data 34 (2) (2005) 657-708, https://doi.org/10.1063/ 1.1796631.

[60] Andrzej Maczynski, Recommended liquid-liquid equilibrium data. Part 1.
Binary alkane-water systems, J. Phys. Chem. Ref. Data 33 (2) (2004) 549-577, https://doi.org/10.1063/1.1643922.

[61] Skrzecz Adam, David G. Shaw, Andrzej Maczynski, Skrzecz Adam, Iupac-nist solubility data series 69 . ternary alcohol-hydrocarbon-water systems, J. Phys. Chem. Ref. Data 28 (4) (1999) 983-1235, https://doi.org/10.1063/1.556052. 